Pacific Journal of Mathematics

CONTROLLED HOMOTOPY TOPOLOGICAL STRUCTURES 


\section{CONTROLLED HOMOTOPY TOPOLOGICAL STRUCTURES}

\section{Bruce Hughes}

Let $p: E \rightarrow B$ be a locally trivial fiber bundle between closed manifolds where $\operatorname{dim} E \geq 5$ and $B$ has a handlebody decomposition. A controlled homotopy topological structure (or a controlled structure, for short) is a map $f: M \rightarrow E$ where $M$ is a closed manifold of the same dimension as $E$ and $f$ is a $p^{-1}(\varepsilon)$-equivalence for every $\varepsilon>0$ (see $\S 2$ ). It is the purpose of this paper to develop an obstruction theory which answers the question: when is $f$ homotopic to a homeomorphism, with arbitrarily small metric control measured in $B$ ? This theory originated with an idea of $W$. C. Hsiang that a controlled structure gives rise to a cross-section of a certain bundle over $B$, associated to the Whitney sum of $p: E \rightarrow B$ and the tangent bundle of $B$.

1. Introduction. In $\S 3$ we define a semi-simplicial complex $\mathscr{S}(p: E \rightarrow B)$, called the space of controlled structures on $p: E \rightarrow B$. Roughly, an $n$-simplex of $\mathscr{S}(p: E \rightarrow B)$ is an $n$-parameter family of controlled structures on $p: E \rightarrow B$. The study of the homotopy relation in $\mathscr{S}(p: E \rightarrow B)$ was initiated in $\left[\mathbf{H}_{1}, \S 8\right]$. For example, if $f: M \rightarrow E$ is a controlled structure, then $f$ is $p^{-1}(\varepsilon)$-homotopic to a homeomorphism for every $\varepsilon>0$ if and only if [f] $=$ [id] in $\pi_{0} \mathscr{S}(p: E \rightarrow B)$. The higher homotopy groups of $\mathscr{S}(p: E \rightarrow B)$ have analogous implications concerning parameterized versions of this problem (see $\S 3$ ). The main objective then is to understand the homotopy type of $\mathscr{S}(p: E \rightarrow B)$. This is accomplished as follows.

Let $\hat{p}: T B \oplus E \rightarrow B$ be the Whitney sum of the tangent bundle of $B$ and $E$. This new bundle has fiber $\mathbf{R}^{m} \times F$ where $m=\operatorname{dim} B$ and $F$ is the fiber of $p: E \rightarrow B$. In $\S 5$ we construct an associated bundle $\tilde{p}: \tilde{E} \rightarrow B$ with fiber $\mathscr{S}\left(\pi: \mathbf{R}^{m} \times F \rightarrow \mathbf{R}^{m}\right)$ where $\pi: \mathbf{R}^{m} \times F \rightarrow \mathbf{R}^{m}$ denotes projection. The main result of this paper is the following theorem.

THEOREM 1. The space of controlled structures $\mathscr{S}(p: E \rightarrow B)$ is homotopy equivalent to the semi-simplicial complex of cross-sections of $\tilde{p}: \tilde{E} \rightarrow B$. 
Corollary 2. The set of path components $\pi_{0} \mathscr{S}(p: E \rightarrow B)$ is in one-to-one correspondence with the set of vertical homotopy classes of cross-sections of $\tilde{p}: \tilde{E} \rightarrow B$.

Under this correspondence the class of the identity on $E$ in $\pi_{0} \mathscr{S}(p: E \rightarrow B)$ corresponds to the vertical homotopy class of a naturally defined zero-section $s: B \rightarrow \tilde{E}$. This cross-section is defined in $\S 5$. Therefore, the following result follows from Corollary 2 and the results from $\left[\mathbf{H}_{1}\right]$ mentioned above (and which are discussed in further detail in $\S 3$ ).

Corollary 3. Associated to every controlled structure $f: M \rightarrow E$ there is a cross-section of the bundle $\tilde{p}: \tilde{E} \rightarrow B$ which is vertically homotopic to the zero-section $s: B \rightarrow \tilde{E}$ if and only if $f$ is $p^{-1}(\varepsilon)$ homotopic to a homeomorphism for every $\varepsilon>0$.

In order to understand the vertical homotopy classes of crosssections of $\tilde{p}: \tilde{E} \rightarrow B$, it is of course necessary to understand the homotopy groups of the fiber $\mathscr{S}\left(\pi: \mathbf{R}^{m} \times F \rightarrow \mathbf{R}^{m}\right)$. This is the subject of the forthcoming paper [HTW].

The study of controlled equivalences between topological manifolds was initiated by Chapman and Ferry $[\mathbf{C F}]$. Chapman $\left[\mathbf{C}_{\mathbf{1}}\right],\left[\mathbf{C}_{\mathbf{2}}\right]$ and Farrell and Hsiang [ $\left.\mathbf{F H}_{1}\right],\left[\mathbf{F H}_{2}\right]$ have studied controlled equivalences into bundles.

Quinn's celebrated work on "ends of maps" $\left[\mathbf{Q}_{1}-\mathbf{Q}_{7}\right]$ deals primarily with controlled simple homotopy theory, controlled pseudo-isotopy theory, and controlled surgery theory. The author's versions of controlled Whitehead spaces and controlled pseudo-isotopy spaces $\left[\mathbf{H}_{3}\right]$ can be shown to be homotopy equivalent to spaces of cross-sections using the methods of the present paper. In this setting the fiber has already been analyzed $\left[\mathbf{H}_{2}\right]$. Quinn has informed the author that this cohomological approach (i.e., the cross-section interpretation) to controlled simple homotopy theory and controlled pseudo-isotopy theory is just "Poincaré dual" to his homological approach and that he worked out the details of this correspondence some time ago. Moreover, Quinn's work is much more general in that the control map $p$ need not be a bundle projection and the control space $B$ need not be a manifold. On the other hand, Quinn's work on controlled pseudoisotopies $\left[\mathbf{Q}_{\mathbf{4}}\right]$ concerns only a single pseudo-isotopy rather than $n$ parameter families of pseudo-isotopies as in $\left[\mathbf{H}_{3}\right]$. Quinn has also 
pointed out that the results in $\left[\mathbf{C}_{2}\right]$ and $\left[\mathbf{F H}_{2}\right]$ on controlled structures can be derived from $\left[\mathbf{Q}_{\mathbf{1}}\right]$.

The proof of Theorem 1 is based on Gromov's immersion theoretic machine as delineated by Siebenmann in [KS, Essay V]. It would be useful for the reader to be familiar with that reference. In addition, the reader would benefit from being familiar with [AH].

The main technical tools on which this work rests are the author's previous results on approximate fibrations $\left[\mathbf{H}_{1}\right]$. Approximate fibrations arise here as follows: if $f: M \rightarrow E$ is a controlled structure, then $p f: M \rightarrow B$ is an approximate fibration.

As mentioned at the beginning of this introduction, this paper is based on an idea of W. C. Hsiang. The author wishes to express his gratitude to Professor Hsiang for explaining to him the correspondence between controlled structures and cross-sections of the associated bundle.

2. Preliminaries. Throughout this paper $p: E \rightarrow B$ will denote a fixed locally trivial fiber bundle with fiber $F$. We assume that $E$ and $B$ are closed manifolds, $E$ has dimension $k \geq 5$, and $B$ has a handlebody decomposition. The handlebody decomposition assumption on $B$ is required because of the use of the results from $\left[\mathbf{H}_{1}\right]$. It is now known that all manifolds except nonsmoothable 4-manifolds have handlebody decompositions $\left[\mathbf{Q}_{3}\right]$. We let $m$ denote the dimension of $B$. We make no assumptions on the fiber $F$. Of course, $\mathbf{R}^{m} \times F$ is a manifold and $F$ is a compact ANR.

We now recall some definitions. Let $f: X \rightarrow Y$ be a proper map (i.e., inverse images of compacta are compact) between locally compact separable metric ANRs and let $\alpha$ be an open cover of $Y$. Then $f$ is an $\alpha$-fibration provided that given any $Z$ and maps $F: Z \times[0,1] \rightarrow Y$ and $g: Z \rightarrow X$ for which $F(z, 0)=f g(z)$ for each $z$ in $Z$, then there exists a map $G: Z \times[0,1] \rightarrow X$ such that $G(z, 0)=g(z)$ for each $z$ and $f G$ is $\alpha$-close to $F$. If $f$ is an $\alpha$-fibration for every open cover $\alpha$ of $Y$, then $f$ is an approximate fibration [CD]. If $\varepsilon>0$, then $\varepsilon$ also denotes the open cover of $Y$ by balls of diameter $\varepsilon$. Thus, we also speak of $\varepsilon$-fibrations.

The proper map $f: X \rightarrow Y$ is said to be an $\alpha$-equivalence (where $\alpha$ is an open cover of $Y$ ) if there exists a proper map $g: Y \rightarrow X$ such that $f g$ is $\alpha$-homotopic to $\operatorname{id}_{Y}$ and $g f$ is $f^{-1}(\alpha)$-homotopic to $\mathrm{id}_{X}$ where the homotopies are proper and $f^{-1}(\alpha)$ denotes the open cover of $X$ defined by $f^{-1}(\alpha)=\left\{f^{-1}(U) \mid U \in \alpha\right\}$. 
In this paper a fiber preserving (f.p.) map is a map which preserves the obvious fibers over an $n$-simplex $\Delta^{n}$. Specifically, if $\rho: X \rightarrow \Delta^{n}$, $\sigma: Y \rightarrow \Delta^{n}$, and $f: X \rightarrow Y$ are maps, then $f$ is f.p. if $\sigma f=\rho$. Usually the maps $\rho$ and $\sigma$ will be understood to be some obvious projections and will not be explicitly mentioned.

3. The space of controlled structures. In this section we define $\mathscr{S}(p: E \rightarrow B)$, the space of controlled structures on $p: E \rightarrow B$. This space is defined as a semi-simplicial complex and the reader is referred to [M] for information about semi-simplicial topology.

A typical $n$-simplex of $\mathscr{S}(p: E \rightarrow B)$ is of the form $f: M \rightarrow E \times \Delta^{n}$ where

(1) $M \subset \mathbf{R}^{s} \times \Delta^{n} \subset \mathbf{R}^{\infty} \times \Delta^{n}$ for some $n$ so that the projection $\mathbf{R}^{\infty} \times \Delta^{n} \rightarrow \Delta^{n}$ restricts to a fibering $M \rightarrow \Delta^{n}$ with closed $k$-manifold fibers,

(2) $f$ is fiber preserving over $\Delta^{n}$,

(3) $f$ is a f.p. $(p \times \text { id })^{-1}(\varepsilon)$-equivalence for every $\varepsilon>0$.

This last condition can be rephrased to say $f$ is a f.p. map such that $f$ is a homotopy equivalence and $\left(p \times\right.$ id) $f: M \rightarrow B \times \Delta^{n}$ is an approximate fibration (see $\left[\mathbf{H}_{2}\right.$, Lemma 2.1]). This fact will be used repeatedly below. Note that $\mathscr{S}(p: E \rightarrow B)$ satisfies the Kan extension condition [M, p.2].

The definition of $\mathscr{S}(p: E \rightarrow B)$ given above differs slightly from the definition given in $\left[\mathbf{H}_{1}, \S 8\right]$. The two different complexes can be shown to be homotopy equivalent using the methods in $\left[\mathbf{H}_{3}, \S 3\right]$. However, we will not use that equivalence here. Instead, we will sketch a proof of an analogue of $\left[\mathbf{H}_{1}\right.$, Theorem 8.1].

For notation, let $f: M \rightarrow E \times \Delta^{n}$ and $g: N \rightarrow E \times \Delta^{n}$ be two $n$-simplices of $\mathscr{S}(p: E \rightarrow B)$ such that $f=g$ over $E \times \partial \Delta^{n}$. If $\rho: M \rightarrow \Delta^{n}$ and $\rho^{\prime}: N \rightarrow \Delta^{n}$ are the given fiberings, then this means that $\rho^{-1}\left(\partial \Delta^{n}\right)=\left(\rho^{\prime}\right)^{-1}\left(\partial \Delta^{n}\right)$ and $f\left|\rho^{-1}\left(\partial \Delta^{n}\right)=g\right| \rho^{-1}\left(\partial \Delta^{n}\right)$.

Proposition 3.1. The simplices $f$ and $g$ are homotopic rel boundary in $\mathscr{S}(p: E \rightarrow B)$ if and only if for every $\varepsilon>0$ there exists a f.p. homeomorphism $h: M \rightarrow N$ such that $h \mid \rho^{-1}\left(\partial \Delta^{n}\right)=\mathrm{id}$ and $g h$ is $f . p$. $(p \times \mathrm{id})^{-1}(\varepsilon)$-homotopic to $f$ rel $\rho^{-1}\left(\partial \Delta^{n}\right)$.

Proof. Suppose first that $f$ and $g$ are homotopic rel boundary in $\mathscr{S}(p: E \rightarrow B)$. This means that there is a map $\tilde{f}: \tilde{M} \rightarrow E \times \Delta^{n} \times[0,1]$ 
where

(1) $\tilde{M} \subset \mathbf{R}^{s} \times \Delta^{n} \times[0,1] \subset \mathbf{R}^{\infty} \times \Delta^{n} \times[0,1]$ for some $s$ so that the projection $\mathbf{R}^{\infty} \times \Delta^{n} \times[0,1] \rightarrow \Delta^{n} \times[0,1]$ restricts to a fibering $\tilde{\rho}: \tilde{M} \rightarrow \Delta^{n} \times[0,1]$ with closed $k$-manifold fibers,

(2) $\tilde{f}$ is a f.p. over $\Delta^{n} \times[0,1]$,

(3) $\tilde{f}$ is a f.p. $(p \times \text { id })^{-1}(\varepsilon)$-equivalence for every $\varepsilon>0$,

(4) $\tilde{p}^{-1}\left(\Delta^{n} \times\{0\}\right)=M \times\{0\}$ and $\tilde{\rho}^{-1}\left(\Delta^{n} \times\{1\}\right)=N \times\{1\}$,

(5) $\tilde{f} \mid M \times\{0\}=f$ and $\tilde{f} \mid N \times\{1\}=g$,

(6) $\tilde{\rho}^{-1}\left(\partial \Delta^{n} \times[0,1]\right)=\rho^{-1}\left(\partial \Delta^{n}\right) \times[0,1]$,

(7) $\tilde{f}\left|\tilde{\rho}^{-1}\left(\partial \Delta^{n} \times[0,1]\right)=f\right| \rho^{-1}\left(\partial \Delta^{n}\right) \times$ id.

Let $k: A \times \Delta^{n} \times[0,1] \rightarrow \tilde{M}$ be a trivializing homeomorphism for $\tilde{\rho}: \tilde{M} \rightarrow \Delta^{n} \times[0,1]$. Note that $A$ is a closed $k$-manifold. Let $q: A \times \Delta^{n} \times$ $[0,1] \rightarrow B \times \Delta^{n} \times[0,1]$ be the composition $q=(p \times \mathrm{id}) \tilde{f} k$. Then $q$ is a f.p. approximate fibration. Let $G: A \times \partial \Delta^{n} \times[0,1] \rightarrow A \times \partial \Delta^{n} \times[0,1]$ be the f.p. homeomorphism given by $G=k^{-1}\left[\left(k \mid\left(A \times \partial \Delta^{n} \times\{0\}\right)\right) \times \mathrm{id}_{[0,1]}\right]$.

Now use [H1, Theorem 7.5] to find a f.p. homeomorphism $H$ : $A \times \Delta^{n} \times[0,1] \rightarrow A \times \Delta^{n} \times[0,1]$ such that $H \mid A \times \Delta^{n} \times\{0\}=\mathrm{id}$, $H \mid A \times \partial \Delta^{n} \times[0,1]=G$ and $q H$ is close to $q \mid\left(A \times \Delta^{n} \times\{0\}\right) \times$ id. Let $h: M \rightarrow N$ be the f.p. homeomorphism given by the composition

$$
\begin{aligned}
M= & \tilde{\rho}^{-1}\left(\Delta^{n} \times\{0\}\right) \stackrel{k^{-1}}{\longrightarrow} A \times \Delta^{n} \times\{0\} \stackrel{\text { id }}{\longrightarrow} A \times \Delta^{n} \times\{1\} \\
& \stackrel{H \mid}{\longrightarrow} A \times \Delta^{n} \times\{1\} \stackrel{k \mid}{\longrightarrow} \tilde{\rho}^{-1}\left(\Delta^{n} \times\{1\}\right)=N .
\end{aligned}
$$

It is not hard to see that $h$ has the desired properties.

For the proof of the converse, we refer the reader to the proof of [H $\mathbf{H}_{1}$, Theorem 8.1].

Corollary 3.2. Let $f: M \rightarrow E$ and $g: N \rightarrow E$ be two controlled structures on $p: E \rightarrow B$, i.e., vertices in $\mathscr{S}(p: E \rightarrow B)$. Then $[f]=[g]$ in $\pi_{0} \mathscr{S}(p: E \rightarrow B)$ if and only if for every $\varepsilon>0$ there exists $a$ homeomorphism $h: M \rightarrow N$ such that gh is $p^{-1}(\varepsilon)$-homotopic to a homeomorphism.

CoRollary 3.3. Let $f: M \rightarrow E$ be a controlled structure on $p$ : $E \rightarrow B$. Then $[f]=\left[\mathrm{id}_{E}\right]$ in $\pi_{0} \mathscr{S}(p: E \rightarrow B)$ if and only if $f$ is $p^{-1}(\varepsilon)$-homotopic to a homeomorphism for every $\varepsilon>0$.

The next result shows that the metric control can be relaxed a bit. (Of course, there are also $n$-parameter versions of this.) 
Corollary 3.4. For every $\varepsilon>0$ there exists a $\delta>0$ so that if $M$ is a closed $k$-manifold and $f: M \rightarrow E$ is a $p^{-1}(\delta)$-equivalence, then there is a well-defined obstruction $\sigma(f)$ in $\pi_{0} \mathscr{S}(p: E \rightarrow B)$ such that $\sigma(f)=\left[\mathrm{id}_{E}\right]$ if and only if $f$ is $p^{-1}(\varepsilon)$-homotopic to a homeomorphism.

Proof. If $\delta$ is small enough, then $p f$ is $\delta^{\prime}$-homotopic to an approximate fibration $\left[\mathbf{C}_{2}\right.$, Theorem 1] where $\delta^{\prime}$ is small. This homotopy can be lifted to show that $f$ is $p^{-1}\left(\delta^{\prime}\right)$-homotopic to a map $g: M \rightarrow E$ such that $p g$ is an approximate fibration. It follows that $g$ is a $p^{-1}(\mu)$-equivalence for every $\mu>0$ and we define $\sigma(f)=[g]$ in $\pi_{0} \mathscr{S}(p: E \rightarrow B)$.

In light of Proposition 3.1, we only have to show tht $\sigma(f)$ is welldefined. To this end suppose that $f$ is also $p^{-1}\left(\delta^{\prime}\right)$-homotopic of $g^{\prime}$ where $g^{\prime}$ is also a $p^{-1}(\mu)$-equivalence for every $\mu>0$. Then $g$ is $p^{-1}\left(2 \delta^{\prime}\right)$-homotopic to $g^{\prime}$. Using the Deformation Theorem of [ $\left.\mathbf{H}_{\mathbf{1}}\right]$ it is easy to deform that homotopy to get a path in $\mathscr{S}(p: E \rightarrow B)$ showing that $[g]=\left[g^{\prime}\right]$.

We will also need the space of controlled structures on the trivial bundle $\pi: \mathbf{R}^{m} \times F \rightarrow \mathbf{R}^{m}$. This semi-simplicial complex will be denoted by $\mathscr{S}\left(\mathbf{R}^{m} \times F\right)$ instead of $\mathscr{S}\left(\pi: \mathbf{R}^{m} \times F \rightarrow \mathbf{R}^{m}\right)$. It is defined in the same manner as $\mathscr{S}(p: E \rightarrow B$ ) except (if $m>0$ ) the fibers of $M \rightarrow \Delta^{n}$ are no longer required to be compact, but they are required to be open $k$-manifolds.

4. Controlled structures over open subsets. In this section we will associate to certain open subsets $U$ of $B$ a Kan semi-simplicial complex $\mathscr{S}(U)$, the space of controlled structures over $U$. This is done in such a way that $\mathscr{S}$ becomes a contravariant functor on the category of these open subsets and inclusion maps. The important sheaf-like properties of this functor are discussed in this section.

Let $N$ be a compact codimension 0 submanifold of $B$ with interior $\dot{N}$. A typical $n$-simplex of $\mathscr{S}(\stackrel{\circ}{N})$ is of the form $f: M \rightarrow p^{-1}(\stackrel{\circ}{N}) \times \Delta^{n}$ where

(1) $M \subset \mathbf{R}^{s} \times \Delta^{n} \subset \mathbf{R}^{\infty} \times \Delta^{n}$ for some $s$ so that the projection $\mathbf{R}^{\infty} \times \Delta^{n} \rightarrow \Delta^{n}$ restricts to a fibering $M \rightarrow \Delta^{n}$ with boundariless $k$ manifold fibers,

(2) $f$ is fiber preserving over $\Delta^{n}$,

(3) $f$ is a homotopy equivalence and $(p \times$ id) $f$ is an approximate fibration.

Note that $\mathscr{S}(B)$ is just the complex $\mathscr{S}(p: E \rightarrow B)$ of the previous section. 
If $N_{1} \subset N_{2}$ are two compact codimension 0 submanifolds of $B$, then there is a restriction map $r: \mathscr{S}\left(\dot{N}_{2}\right) \rightarrow \mathscr{S}\left(\dot{N}_{1}\right)$ defined as follows: if $f: M \rightarrow p^{-1}\left(\dot{N}_{2}\right) \times \Delta^{n}$ is an $n$-simplex of $\mathscr{S}\left(\dot{N}_{2}\right)$, then $r(f)$ is $f \mid$ : $f^{-1}\left(p^{-1}\left(\dot{N}_{1}\right) \times \Delta^{n}\right) \rightarrow p^{-1}\left(\dot{N}_{1}\right) \times \Delta^{n}$. The only problem with this definition is that it is conceivable that the map $f^{-1}\left(p^{-1}\left(\dot{N}_{1}\right) \times \Delta^{n}\right) \rightarrow$ $\Delta^{n}$ is not a bundle projection. However, the next lemma takes care of this problem. This is the reason why $\mathscr{S}$ was defined only over interiors of compact manifolds.

LEMMA 4.1. Let $N$ be a compact manifold and let $g: M \rightarrow \stackrel{\circ}{N} \times \Delta^{n}$ be a proper map such that the composition $\pi: M \stackrel{g}{\longrightarrow} \stackrel{ }{N} \times \Delta^{n} \stackrel{\text { proj }}{\longrightarrow} \Delta^{n}$ is a submersion whose fibers are manifolds without boundary, of dimension $\geq$ 5. If $g \mid: \pi^{-1}(t) \rightarrow \stackrel{\circ}{N}$ is an approximate fibration for each $t$ in $\Delta^{n}$, then $\pi$ is a bundle projection.

Proof. Since $\partial N$ is collared $N$, we can consider $\mathbf{R} \times \partial N$ as an open subset of $\dot{N}$ so that $\dot{N} \backslash(\mathbf{R} \times \partial N)$ is compact. Use [KS, Theorem 1.1, p. 60] to conclude that $\pi \mid: g^{-1}\left(\mathbf{R} \times \partial N \times \Delta^{n}\right) \rightarrow \Delta^{n}$ is a bundle projection. The engulfing condition needed to apply [KS] comes from $\left[\mathbf{C}_{2}\right.$, Lemma 3.4]. Finally, use [S, Lemma 6.9] to conclude that $\pi$ is a bundle projection.

By passing to germs, $\mathscr{S}$ can be extended to compact subsets of $B$. In other words, if $X$ is a compact subset of $B$, then $\mathscr{S}(X)$ is the direct limit of all $\mathscr{S}(\stackrel{\circ}{N})$ where $\stackrel{\circ}{N}$ is the interior of a compact codimension 0 submanifold of $B$ such that $X \subset \dot{N}$. If $Y \subset X$ are compact subsets of $B$, then there is a restriction map $r: \mathscr{S}(X) \rightarrow \mathscr{S}(Y)$. The following propositions record the most important properties of $\mathscr{S}$. The proof of the first one is left to the reader.

Proposition 4.2. If $X$ and $Y$ are compact subsets of $B$, then the following diagram of restriction maps is a fiber homotopy product.

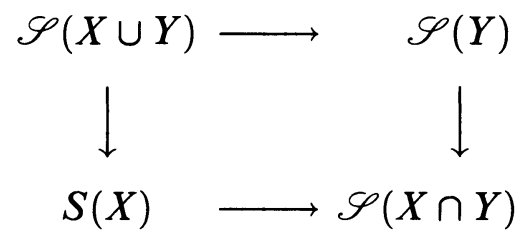

Proposition 4.3. If $Y \subset X$ are compact subsets of $B$, then the restriction $r: \mathscr{S}(X) \rightarrow \mathscr{S}(Y)$ is a Kan fibration. 
Proof. First of all we are given an $(n+1)$-simplex of $\mathscr{S}(Y)$ which is represented by an $(n+1)$-simplex of $\mathscr{S}(V)$ where $Y \subset V$ and $V$ is the interior of a compact codimension 0 submanifold of $B$. Let $g: N \rightarrow p^{-1}(V) \times \Delta^{n} \times[0,1]$ be this $(n+1)$-simplex of $\mathscr{S}(V)$ where we have identified $\Delta^{n+1}$ with $\Delta^{n} \times[0,1]$ and $N \subset \mathbf{R}^{\infty} \times \Delta^{n} \times[0,1]$.

Then we are given a lift of the 0-level of this simplex to an $n$ simplex of $\mathscr{S}(X)$. Thus there is an $n$-simplex $f: M \rightarrow p^{-1}(U) \times \Delta^{n}=$ $p^{-1}(U) \times \Delta^{n} \times\{0\}$ of $\mathscr{S}(U)$ where $X \subset U, U$ is the interior of a compact codimension 0 submanifold of $B$, and $M \subset \mathbf{R}^{\infty} \times \Delta^{n} \times\{0\} \subset$ $\mathbf{R}^{\infty} \times \Delta^{n} \times[0,1]$. Since $f$ is a lift of $g_{0}$ we can assume (after passing to smaller neighborhoods) that $V \subset U$ and $f=g$ over $p^{-1}(V) \times \Delta^{n} \times\{0\}$. Our task is to extend $f$ in an appropriate manner.

Since the maps $N \rightarrow \Delta^{n} \times[0,1]$ and $M \rightarrow \Delta^{n} \times\{0\}$ are bundle projections we can make identifications $N=N^{\prime} \times \Delta^{n} \times[0,1]$ and $M=M^{\prime} \times \Delta^{n} \times\{0\}$ where $M^{\prime} \subset N^{\prime}$. Choose compacta $Y_{i}, i=1,2,3$, such that $Y \subset$ int $Y_{1} \subset Y_{1} \subset$ int $Y_{2} \subset \cdots \subset Y_{3} \subset V$, and choose $\varepsilon>0$ small. By $\left[\mathbf{H}_{1}\right.$, Theorem 7.1] there is a f.p. (over $\Delta^{n} \times[0,1]$ ) homeomorphism $H: N^{\prime} \times \Delta^{n} \times[0,1] \rightarrow N^{\prime} \times \Delta^{n} \times[0,1]$ such that $H_{0}=$ id and $\left(p g_{0} \times\right.$ id $) H$ is $\varepsilon$-close to $(p \times$ id $) g$. Let $\mu: M^{\prime} \times \Delta^{n} \rightarrow[0,1]$ be a map such that

$$
\mu^{-1}(0)=\left(M^{\prime} \times \Delta^{n}\right) \backslash f^{-1}\left(p^{-1}\left(\text { int } Y_{3}\right) \times \Delta^{n}\right)
$$

and

$$
\mu^{-1}(1)=f^{-1}\left(p^{-1}\left(Y_{2}\right) \times \Delta^{n}\right) .
$$

Then define $\tilde{f}: M^{\prime} \times \Delta^{n} \times[0,1] \rightarrow p^{-1}(U) \times \Delta^{n} \times[0,1]$ by setting

$$
\tilde{f}(x, y, z)= \begin{cases}\left(p_{E} g H^{-1}(x, y, \mu(x, y) \cdot z), y, z\right) & \text { if } \mu(x, y) \cdot z>0 \\ (f(x, y), z) & \text { if } \mu(x, y) \cdot z=0\end{cases}
$$

Here $p_{E}$ denotes projection to $E$. One can verify that
(1) $\tilde{f}_{0}=f$,
(2) $\tilde{f}$ is $(p \times \text { id })^{-1}(\varepsilon)$-close to $f \times$ id,
(3) $\tilde{f}=g H^{-1}$ over $p^{-1}\left(Y_{1}\right) \times \Delta^{n} \times[0,1]$.

Unfortunately $(p \times$ id) $\tilde{f}$ need not be an approximate fibration. However, one can use $\left[\mathbf{H}_{1}\right.$, Theorem 5.1] to find an f.p. map $\hat{f}: M^{\prime} \times$ $\Delta^{n} \times[0,1] \rightarrow p^{-1}(U) \times \Delta^{n} \times[0,1]$ such that $\hat{f}_{0}=\tilde{f}_{0}, \hat{f}=\tilde{f}$ over $p^{-1}(Y) \times \Delta^{n} \times[0,1]$ and $(p \times$ id $) \hat{f}$ is an approximate fibration.

Finally, use the Isotopy Extension Theorem [EK, Corollary 1.2] to find a f.p. homeomorphism $\tilde{H}: M^{\prime} \times \Delta^{n} \times[0,1] \rightarrow M^{\prime} \times \Delta^{n} \times[0,1]$ such that $\tilde{H}_{0}=\mathrm{id}$ and $\tilde{H}=H$ on $g^{-1}\left(p^{-1}(Y) \times \Delta^{n} \times[0,1]\right)$. Then the 
map $f^{*}: M^{\prime} \times \Delta^{n} \times[0,1] \rightarrow p^{-1}(U) \times \Delta^{n} \times[0,1]$ defined by $f^{*}: \hat{f} \tilde{H}$ is the required lift of $g$.

It might be worth noting that the proof of the proposition above can be used to prove a homotopy extension property for approximate fibrations. Although we will not need this result, we state it for the interested reader.

CoRollary 4.4. Let $M$ and $N$ be closed manifolds (with $\operatorname{dim} M \geq 5$ and $N$ a handlebody) and let $X \subset U \subset N$ where $X$ is compact and $U$ is open. Let $f: M \rightarrow N$ be an approximate fibration such that $f \mid: f^{-1}(U) \rightarrow U$ extends to a homotopy of approximate fibrations $F: f^{-1}(U) \times[0,1] \rightarrow U \times[0,1]$. Then there exists a homotopy of approximate fibrations $G: M \times[0,1] \rightarrow N \times[0,1]$ such that $G_{0}=f$ and $G\left|f^{-1}(X) \times[0,1]=F\right|$.

The final proposition of this section shows that certain restrictions are homotopy equivalences.

Proposition 4.5. If $X$ is a simplex in $B$ (linear in some chart) and $x \in X$, then the restriction $r: \mathscr{S}(X) \rightarrow \mathscr{S}(x)$ is a homotopy equivalence.

Proof. We will show that $r_{*}: \pi_{n} \mathscr{S}(X) \rightarrow \pi_{n} \mathscr{S}(x)$ is an isomorphism for each $n \geq 0$. The basic geometric fact which makes this true is that there are isotopies of $B$ which shrink $X$ arbitrarily close to $x$.

Let $C$ denote an open chart in $B$ which linearly contains $X$. In order to show that $r_{*}$ is surjective, let $f: M \rightarrow p^{-1}(U) \times \Delta^{n}$ represent an element of $\pi_{n} \mathscr{S}(x)$ where $U$ is the interior of a compact codimension 0 submanifold of $C$ which contains $x$. Let $V$ be an open subset of $B$ containing $x$ such that $\operatorname{cl}(V) \subset U$. Let $h: B \rightarrow B$ be a homeomorphism such that $h \mid V=\mathrm{id}, h$ is compactly supported in $C$, and $X \subset h(U)$. Since the bundle $p: E \rightarrow B$ is trivial over $C$, it is easy to construct a homeomorphism $\tilde{h}: E \rightarrow E$ such that $p \tilde{h}=h p$ and $\tilde{h} \mid p^{-1}(V)=$ id. Then the composition $(\tilde{h} \times \mathrm{id}) f: M \rightarrow p^{-1}(h(U)) \times \Delta^{n}$ defines an element of $\pi_{n} \mathscr{S}(X)$ whose image under $r_{*}$ is $[f]$. The reader should observe that $(\tilde{h} \times \mathrm{id}) f$ has the required control because $\tilde{h}$ covers a homeomorphism of $B$.

In order to show that $r_{*}$ is injective, let $f: M \rightarrow p^{-1}(U) \times \Delta^{n}$ and $g: N \rightarrow p^{-1}(U) \times \Delta^{n}$ represent elements of $\pi_{n} \mathscr{S}(X)$ where $U$ is as above except now $X \subset U$. If $f=g$ in $\pi_{n} \mathscr{S}(x)$, then there is an interior 
$W$ of a compact codimension 0 submanifold of $U$ which contains $x$ and an $(n+1)$-simplex in $\mathscr{S}(W)$ which connects the restrictions of $f$ and $g$. Let $h_{t}: B \rightarrow B, 0 \leq t \leq 1$, be an isotopy supported in $U$ such that $h_{0}=$ id and $X \subset h_{1}(W)$. Construct an isotopy $\tilde{h}_{t}: E \rightarrow E$, $0 \leq t \leq 1$, covering $h_{t}$ such that $\tilde{h}_{0}=\mathrm{id}$.

Then $\left(\tilde{h}_{1} \times\right.$ id $) f$ and $\left(\tilde{h}_{1} \times\right.$ id $) g$ are equal in $\pi_{n} \mathscr{S}(X)$. Using the isotopy $\tilde{h}_{t}$, it is easy to see that $f$ equals $\left(\tilde{h}_{1} \times\right.$ id) $f$ and $g$ equals $\left(\tilde{h}_{1} \times\right.$ id $) g$ in $\pi_{n} \mathscr{S}(X)$.

5. An associated bundle and its space of cross-sections. In this section we construct the bundle $\tilde{p}: \tilde{E} \rightarrow B$ whose space of cross-sections turns out to be homotopy equivalent to $\mathscr{S}(p: E \rightarrow B)$. We will use the terminology of [M, Chapter IV].

For notational simplicity, we will assume in this section that $B$ is a polyhedron with a fixed triangulation. Thus, we can think of $B$ as a semi-simplicial complex with one non-degenerate $n$-simplex for each $n$-simplex of $B$. If $B$ happens to be non-polyhedral, then we could achieve the same end by replacing $B$ by its singular complex.

Recall that $F$ is the fiber of the bundle $p: E \rightarrow B$. Thus, the group of the bundle is $\mathscr{H}(F)$, the space of all homeomorphisms of $F$ onto $F$.

Fix a topological tangent bundle of $B, p_{1}: T B \rightarrow B$, where $T B \subset$ $B \times B$ is a neighborhood of the diagonal and $p_{1}$ is projection onto the first coordinate. The fiber of this bundle is $\mathbf{R}^{m}$ and the group is $\mathrm{TOP}_{m}$, the space of homeomorphisms of $\mathbf{R}^{m}$ onto $\mathbf{R}^{m}$ which fix the origin.

For each simplex $\sigma$ of $B$, fix local trivializations $h_{\sigma}^{1}: \sigma \times \mathbf{R}^{m} \rightarrow$ $p_{1}^{-1}(\sigma)$ and $h_{\sigma}^{2}: \sigma \times F \rightarrow p^{-1}(\sigma)$. By first defining these over vertices and then inductively working up the dimensions of the simplices of $B$, we can achieve the regularity conditions $[\mathbf{M}, \mathrm{p} .77]$ which state that the compositions

$$
\partial_{i} \sigma \times \mathbf{R}^{m} \stackrel{h_{\sigma}^{1} l}{\longrightarrow} p_{1}^{-1}\left(\partial_{i} \sigma\right) \stackrel{\left(h_{\partial_{l}}^{1}\right)^{-1}}{\longrightarrow} \partial_{i} \sigma \times \mathbf{R}^{m}
$$

and

$$
\partial_{i} \sigma \times F \stackrel{h_{\sigma}^{2} \mid}{\longrightarrow} p^{-1}\left(\partial_{i} \sigma\right) \stackrel{\left(h_{\partial_{1}}^{2}\right)^{-1}}{\longrightarrow} \partial_{i} \sigma \times F
$$

are the identities whenever $i>0$ and $\operatorname{dim} \sigma>0$. For all of this it is important to have the vertices of $B$ ordered.

Form the Whitney sum $\hat{p}: T B \oplus E \rightarrow B$ of $p_{1}: T B \rightarrow B$ and $p:$ $E \rightarrow B$. This bundle has fiber $\mathbf{R}^{m} \times F$ and group $G=\mathrm{TOP}_{m} \oplus \mathscr{H}(F)$. We also think of $G$ as the space TOP $m \times \mathscr{H}(F)$. 
We have local trivializations $h_{\sigma}: \sigma \times \mathbf{R}^{m} \times F \rightarrow \hat{p}^{-1}(\sigma)$ induced by $h_{\sigma}^{1}$ and $h_{\sigma}^{2}$. These satisfy the regularity condition which states that the composition

$$
\partial_{i} \sigma \times \mathbf{R}^{m} \times F \stackrel{h_{\sigma} \mid}{\longrightarrow} \hat{p}^{-1}\left(\partial_{i} \sigma\right) \stackrel{\left(h_{\partial_{1}} \sigma\right)^{-1}}{\longrightarrow} \partial_{i} \sigma \times \mathbf{R}^{m} \times F
$$

is the identity whenever $i>0$ and $\operatorname{dim} \sigma>0$.

Define transformation elements [M, p. 76] as follows. If $\sigma$ is a simplex of $B$ with $\operatorname{dim} \sigma=q>0$, let $\tau(\sigma): \partial_{0} \sigma \times \mathbf{R}^{m} \times F \rightarrow \partial_{0} \sigma \times$ $\mathbf{R}^{m} \times F$ be the homeomorphism (which is f.p. over $\partial_{0} \sigma$ ) given by the composition

$$
\partial_{0} \sigma \times \mathbf{R}^{m} \times F \stackrel{h_{\sigma} \mid}{\longrightarrow} \hat{p}^{-1}\left(\partial_{0} \sigma\right) \stackrel{\left(h_{\partial_{0}} \sigma\right)^{-1}}{\longrightarrow} \partial_{0} \sigma \times \mathbf{R}^{m} \times F .
$$

If $X$ is a space, let Sing $X$ denote the singular complex of $X$. Then Sing $G=\operatorname{Sing} \operatorname{TOP}_{m} \times \operatorname{Sing} \mathscr{H}(F)$ is a semi-simplicial group. And $\tau$ defines a function, called a twisting function $[\mathbf{M}$, p. 71$], \tau:[B]_{q} \rightarrow$ [Sing $G]_{q-1}$, where $[\cdot]_{q}$ denotes the set of $q$-simplices of a semisimplicial complex. It is important to note that the image of $\tau$ lies in Sing $G$ and not just in Sing $\mathscr{H}\left(\mathbf{R}^{m} \times F\right)$.

Recall from $\S 3$ that $\mathscr{S}\left(\mathbf{R}^{m} \times F\right)$ denotes the space of controlled structures on the trivial bundle $\pi: \mathbf{R}^{m} \times F \rightarrow \mathbf{R}^{m}$. Then Sing $G$ acts (semi-simplicially) on $\mathscr{S}\left(\mathbf{R}^{m} \times F\right)$ by composition. That is, if $f: M \rightarrow \mathbf{R}^{m} \times F \times \Delta^{n}$ is an $n$-simplex of $\mathscr{S}\left(\mathbf{R}^{m} \times F\right)$ and $g: \mathbf{R}^{m} \times$ $F \times \Delta^{n} \rightarrow \mathbf{R}^{m} \times F \times \Delta^{n}$ is an $n$-simplex of Sing $G$, then $g \cdot f$ is defined to be the composition $g \circ f$. Note that Sing $\mathscr{C}\left(\mathbf{R}^{m} \times F\right)$ does not act on $\mathscr{S}\left(\mathbf{R}^{m} \times F\right)$ by composition.

We now observe that $\tau$ satisfies the four properties required of a twisting function [M, p. 71].

LEMMA 5.1. The function $\tau$ satisfies the following properties:

(1) $\partial_{0} \tau(\sigma)=\left[\tau\left(\partial_{0} \sigma\right)\right]^{-1} \tau\left(\partial_{1} \sigma\right)$ for each $\sigma$,

(2) $\partial_{i} \tau(\sigma)=\tau\left(\partial_{i+1} \sigma\right)$ for each $\sigma$ and $i>0$,

(3) $s_{i} \tau(\sigma)=\tau\left(s_{i+1} \sigma\right)$ for each $\sigma$ and $i \geq 0$,

(4) $\tau\left(s_{0} \sigma\right)=$ id for each $\sigma$.

Proof. Property 1 follows by writing down the homeomorphisms, using the regularity conditions and the fact that $\partial_{0} \partial_{0}=\partial_{0} \partial_{1}$.

Property 2 follows from regularity and the fact that $\partial_{0} \partial_{i+1}=\partial_{i} \partial_{0}$. Properties 3 and 4 are immediate.

We can now define a twisted Cartesian product [M, p. 71], denoted $B \times{ }_{\tau} \mathscr{S}\left(\mathbf{R}^{m} \times F\right)$, with fiber $\mathscr{S}\left(\mathbf{R}^{m} \times F\right)$, base $B$, and group Sing $G$ as 
follows:

(1) $\left[B \times{ }_{\tau} \mathscr{S}\left(\mathbf{R}^{m} \times F\right)\right]_{q}=[B]_{q} \times\left[\mathscr{S}\left(\mathbf{R}^{m} \times F\right)\right]_{q}$,

(2) $\partial_{i}(\sigma, f)=\left(\partial_{i} \sigma, \partial_{i} f\right)$ for $i>0$,

(3) $\partial_{0}(\sigma, f)=\left(\partial_{0} \sigma, \tau(\sigma) \cdot \partial_{0} f\right)$,

(4) $s_{i}(\sigma, f)=\left(s_{i} \sigma, s_{i} f\right)$ for $i \geq 0$.

Let $\tilde{E}=B \times_{\tau} \mathscr{S}\left(\mathbf{R}^{m} \times F\right)$ and define $\tilde{p}: \tilde{E} \rightarrow B$ by $\tilde{p}(\sigma, f)=\sigma$. (Note that our base and fiber occur in reverse order from those in [M].)

Let $\Gamma(\tilde{E})$ denote the semi-simplicial complex of cross-sections of $\tilde{p}: \tilde{E} \rightarrow B$. Thus, a typical $n$-simplex of $\Gamma(\tilde{E})$ is a semi-simplicial map $\gamma: B \times \Delta^{n} \rightarrow \tilde{E}$ such that $\tilde{p} \gamma(\sigma, \rho)=\sigma$ whenever $(\sigma, \rho)=d$ is a $q$-simplex of $B \times \Delta^{n}$. We are of course thinking of $B \times \Delta^{n}$ as the product of two semi-simplicial complexes. Thus, $\sigma$ is a $q$-simplex of $B$ and $\rho$ is a $q$-simplex of $\Delta^{n}$. And $\gamma(d)=\left(\sigma, f_{d}\right) \in B \times{ }_{\tau} \mathscr{S}\left(\mathbf{R}^{m} \times F\right)$ where $f_{d}: M_{d} \rightarrow \mathbf{R}^{m} \times F \times \Delta^{q}$ is a $q$-simplex of $\mathscr{S}\left(\mathbf{R}^{m} \times F\right)$.

The zero-section $s: B \rightarrow \tilde{E}$ mentioned in the introduction is defined by $s(\sigma)=(\sigma, \mathrm{id})$.

For every $q$-simplex $d=(\sigma, \rho)$ in $B \times \Delta^{n}$ there is a preferred "linear" map $e_{d}: \Delta^{q} \rightarrow B \times \Delta^{n}$ such that $e_{d}\left(\Delta^{q}\right)=d$ and so that the following diagram commutes:

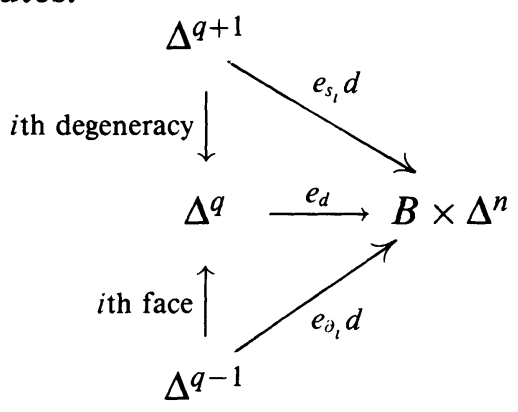

Define an embedding $\tilde{e}_{d}: M_{d} \rightarrow \mathbf{R}^{\infty} \times B \times \Delta^{n}$ by the composition $M_{d} \subset \mathbf{R}^{\infty} \times \Delta^{q} \stackrel{\text { id }}{\longrightarrow} e_{d} \mathbf{R}^{\infty} \times B \times \Delta^{n}$. Let $\tilde{M}=\bigcup\left\{\tilde{e}_{d}\left(M_{d}\right) \mid d\right.$ is a simplex of $\left.B \times \Delta^{n}\right\}$. Define $\tilde{f}: \tilde{M} \rightarrow T B \oplus E \times \Delta^{n}$ so that the following diagram commutes for each simplex $d^{q}=(\sigma, \rho)$ of $B \times \Delta^{n}$ :

$$
\begin{gathered}
\tilde{M} \stackrel{\tilde{f}}{\longrightarrow} T B \oplus E \times \Delta^{n} \supset \hat{p}^{-1}(\sigma) \times \Delta^{n} \\
\tilde{e}_{d}^{-1} \downarrow \\
M_{d} \stackrel{f_{d}}{\longrightarrow} \mathbf{R}^{m} \times F \times \Delta^{q} \stackrel{\text { id } \times e_{d}}{\longrightarrow} \mathbf{R}^{m} \times F \times \sigma \times \Delta^{n}
\end{gathered}
$$

It is not too difficult to see that $\tilde{f}$ is well-defined.

Note that the composition $\tilde{M} \stackrel{\tilde{f}}{\longrightarrow} T B \oplus E \times \Delta^{n} \stackrel{\hat{p} \times \text { id }}{\longrightarrow} B \times \Delta^{n}$ is the restriction of the projection $\mathbf{R}^{\infty} \times B \times \Delta^{n} \rightarrow B \times \Delta^{n}$ and it is a bundle 
projection with open $k$-manifold fibers. Thus, we have associated to the cross-section $\gamma: B \times \Delta^{n} \rightarrow \tilde{E}$ a diagram

$$
\begin{gathered}
\mathbf{R}^{\infty} \times B \times \Delta^{n} \supset \tilde{M} \stackrel{\tilde{f}}{\longrightarrow} T B \oplus E \times \Delta^{n} \\
\qquad \underset{\hat{p} \times \text { id }}{\longrightarrow}{ }_{B \times \Delta^{n}}
\end{gathered}
$$

Define a semi-simplicial complex $\tau \mathscr{S}(p: E \rightarrow B)$, called the space of tangentially controlled structures on $p: E \rightarrow B$ as follows. A typical $n$-simplex is given by a diagram

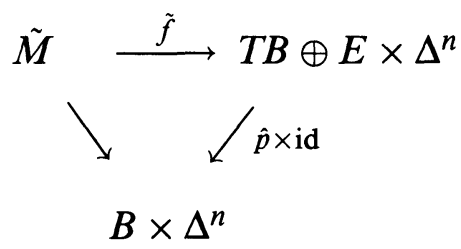

where

(1) $\tilde{M} \subset \mathbf{R}^{s} \times B \times \Delta^{n} \subset \mathbf{R}^{\infty} \times B \times \Delta^{n}$ for some $s$ so that the projection $\mathbf{R}^{\infty} \times B \times \Delta^{n} \rightarrow B \times \Delta^{n}$ restricts to a fibering $\tilde{M} \rightarrow B \times \Delta^{n}$ with open $k$-manifold fibers,

(2) $\tilde{f}$ is fiber preserving over $B \times \Delta^{n}$ (which of course means that the diagram above commutes),

(3) for each $(b, t)$ in $B \times \Delta^{n}$, the map $\tilde{f} \mid: \tilde{M}_{(b, t)} \rightarrow \hat{p}^{-1}(b) \times\{t\}$ is a $\pi_{b}^{-1}(\alpha)$-equivalence for each open cover $\alpha$ of $p_{1}^{-1}(b)$ where $\pi_{b}$ : $\hat{p}^{-1}(b)=p_{1}^{-1}(b) \times p^{-1}(b) \rightarrow p_{1}^{-1}(b)$ denotes projection and $\tilde{M}_{(b, t)}=$ $\tilde{M} \cap \mathbf{R}^{\infty} \times\{(b, t)\}$.

The construction above defines a semi-simplicial map $\Gamma(\tilde{E}) \rightarrow$ $\tau \mathscr{S}(p: E \rightarrow B)$ which is clearly an isomorphism.

6. Passage to germs. In this section we show that no information is lost when a tangentially controlled structure $f: M \rightarrow T B \oplus E$ is restricted over a smaller tangent bundle neighborhood $T^{\prime} B$ of $\Delta B$ in $B \times B$. Recall from the previous section that the space $\tau \mathscr{S}(p: E \rightarrow B)$ of tangentially controlled structures is just the space of cross-sections of a bundle $\tilde{p}: \tilde{E} \rightarrow B$ with fiber $\mathscr{S}\left(\mathbf{R}^{m} \times F\right)$. Our goal is to show that $\tilde{p}: \tilde{E} \rightarrow B$ is fiber homotopy equivalent to a bundle with fiber $\mathscr{G S}\left(\mathbf{R}^{m} \times F\right)$, the space of germs about the origin of controlled structures on $\mathbf{R}^{m} \times F$.

The formal definition of $\mathscr{G S}\left(\mathbf{R}^{m} \times F\right)$ as a semi-simplicial complex is as follows. A typical $n$-simplex is an equivalence class $\bar{f}$ represented 
by

$$
M \stackrel{f}{\longrightarrow} T \times F \times \Delta^{n} \stackrel{\pi \times \text { id }}{\longrightarrow} T \times \Delta^{n}
$$

where

(1) $T$ is a neighborhood of the origin in $\mathbf{R}^{m}$ homeomorphic to $\mathbf{R}^{m}$,

(2) $M \subset \mathbf{R}^{s} \times \Delta^{n} \subset \mathbf{R}^{\infty} \times \Delta^{n}$ for some $s$ so that the projection $\mathbf{R}^{\infty} \times \Delta^{n} \rightarrow \Delta^{n}$ restricts to a fibering $M \rightarrow \Delta^{n}$ with open $k$-manifold fibers,

(3) $f$ is fiber preserving over $\Delta^{n}$,

(4) $f$ is a homotopy equivalence and $(\pi \times \mathrm{id}) f$ is an approximate fibration where $\pi: T \times F \rightarrow T$ is projection.

Another such situation, denoted by $f^{\prime}$ and given by

$$
M^{\prime} \stackrel{f^{\prime}}{\longrightarrow} T^{\prime} \times F \times \Delta^{n} \stackrel{\pi \times \mathrm{id}}{\longrightarrow} T^{\prime} \times \Delta^{n},
$$

is equivalent to $f$ provided there is a neighborhood $T^{\prime \prime}$ of the origin such that $T^{\prime \prime} \subset T \cap T^{\prime}$ and $f=f^{\prime}$ over $T^{\prime \prime} \times F \times \Delta^{n}$.

We will show in Proposition 6.6 below that the natural map $\gamma$ : $\mathscr{S}\left(\mathbf{R}^{m} \times F\right) \rightarrow \mathscr{G S}\left(\mathbf{R}^{m} \times F\right)$, which takes an $n$-simplex $f$ of $\mathscr{S}\left(\mathbf{R}^{m} \times F\right)$ to its germ $\bar{f}$ in $\mathscr{G S}\left(\mathbf{R}^{m} \times F\right)$, is a homotopy equivalence. We first need some preliminary results.

LEMMA 6.1. Let $f: M \rightarrow \mathbf{R}^{m} \times F \times \Delta^{n}$ and $g: N \rightarrow \mathbf{R}^{m} \times F \times \Delta^{n}$ be $n$-simplices of $\mathscr{S}\left(\mathbf{R}^{m} \times F\right)$ which determine the elements $[f]$ and $[g]$ of $\pi_{n} \mathscr{S}\left(\mathbf{R}^{m} \times F\right)$. Then $[f]=[g]$ if and only if for every $\varepsilon>0$ there exists a f.p. homeomorphism $h: M \rightarrow N$ such that $h \mid \rho^{-1}\left(\partial \Delta^{n}\right)=\mathrm{id}$ and $g h$ is f.p. $(\pi \times \mathrm{id})^{-1}(\varepsilon)$-homotopic to $f$ rel $\rho^{-1}\left(\partial \Delta^{n}\right)$.

Remarks on Proof. The proof is essentially the same as the proof of Proposition 3.1. It is understood that $\mathbf{R}^{m}$ is given the standard metric. One only needs to check that the relevant results from $\left[\mathbf{H}_{1}\right]$ hold in this context (cf. $\left[\mathbf{H}_{2}, \S 3\right]$ ).

The proof of the following lemma proceeds along the same lines as the proof of Lemma 6.1 and is left to the reader. See also $\left[\mathbf{H}_{2}\right.$, Proposition 3.4].

LEMMA 6.2. There exists an $\varepsilon>0$ such that if $f: M \rightarrow \mathbf{R}^{m} \times F \times \Delta^{n}$ and $g: N \rightarrow \mathbf{R}^{m} \times F \times \Delta^{n}$ are $n$-simplices of $\mathscr{S}\left(\mathbf{R}^{m} \times F\right)$ with the following properties:

(1) $f=g$ over $\left(\mathbf{R}^{m} \times F \times \partial \Delta^{n}\right) \cup\left(B_{2}^{m} \times F \times \Delta^{n}\right)$,

(2) there is a f.p. homeomorphism $h: M \rightarrow N$ such that $h=\mathrm{id}$ on $f^{-1}\left(\mathbf{R}^{m} \times F \times \partial \Delta^{n}\right) \cup f^{-1}\left(B_{2}^{m} \times F \times \Delta^{n}\right)$, 
(3) gh is f.p. $(\pi \times \text { id })^{-1}(\varepsilon)$-homotopic to $f$ rel $f^{-1}\left(\mathbf{R}^{m} \times F \times \partial \Delta^{n}\right) \cup$ $f^{-1}\left(B_{2}^{m} \times F \times \Delta^{n}\right)$,

then there exist a manifold $\tilde{M} \subset \mathbf{R}^{\infty} \times \Delta^{n} \times[0,1]$ and a map $\tilde{f}: \tilde{M} \rightarrow$ $\mathbf{R}^{m} \times F \times \Delta^{n} \times[0,1]$ such that

(1) $\tilde{f}=f$ over $\mathbf{R}^{m} \times F \times \Delta^{n} \times\{0\}$,

(2) $\tilde{f}=g$ over $\mathbf{R}^{m} \times F \times \Delta^{n} \times\{1\}$,

(3) $\tilde{f}=f \times$ id $=g \times$ id over $\left(\mathbf{R}^{m} \times F \times \partial \Delta^{n} \times[0,1]\right) \cup\left(B_{1}^{m} \times F \times\right.$ $\left.\Delta^{n} \times[0,1]\right)$,

(4) the projection $\mathbf{R}^{\infty} \times \Delta^{n} \times[0,1] \rightarrow \Delta^{n} \times[0,1]$ restricts to a fibering $\tilde{M} \rightarrow \Delta^{n} \times[0,1]$ with open $k$-manifold fibers,

(5) $\tilde{f}$ is fiber preserving over $\Delta^{n} \times[0,1]$,

(6) $\tilde{f}$ is a homotopy equivalence and $(\pi \times \mathrm{id}) \tilde{f}$ is an approximate fibration.

The following lemma contains the key engulfing idea which is needed to show that $\gamma$ is a homotopy equivalence. For notation, we are given $n$-simplices, $f: M \rightarrow \mathbf{R}^{m} \times F \times \Delta^{n}$ and $g: N \rightarrow \mathbf{R}^{m} \times F \times \Delta^{n}$, of $\mathscr{S}\left(\mathbf{R}^{m} \times F\right)$ such that $f=g$ over $\mathbf{R}^{m} \times F \times \partial \Delta^{n}$. We continue to let $\rho^{-1}\left(\partial \Delta^{n}\right)=f^{-1}\left(\mathbf{R}^{m} \times F \times \partial \Delta^{n}\right)$ and to let $\pi: \mathbf{R}^{m} \times F \rightarrow \mathbf{R}^{m}$ denote projection.

LEMMA 6.3. For every $\varepsilon>0$ there exists a $\delta>0$ so that if $h: M \rightarrow N$ is a f.p. map such that

(1) $h \mid \rho^{-1}\left(\partial \Delta^{n}\right)=\mathrm{id}$,

(2) $(\pi \times \mathrm{id}) g h$ is $\delta$-close to $f$,

(3) $h$ is a homeomorphism over $g^{-1}\left(B_{3}^{m} \times F \times \Delta^{n}\right)$,

then $h$ is $f . p((\pi \times \mathrm{id}) g)^{-1}$ (E)-homotopic rel $\rho^{-1}\left(\partial \Delta^{n}\right) \cup f^{-1}\left(B_{1}^{m} \times\right.$ $\left.F \times \Delta^{n}\right) \cup f^{-1}\left(\left(\mathbf{R}^{m} \backslash B_{7}^{m}\right) \times F \times \Delta^{n}\right)$ to a map $\tilde{h}: M \rightarrow N$ which is a homeomorphism over $g^{-1}\left(B_{5}^{m} \times F \times \Delta^{n}\right)$.

Proof. First note that by making a small adjustment to $h$, we may additionally assume that there is a neighborhood $A$ of $\partial \Delta^{n}$ in $\Delta^{n}$ such that $h$ is a homeomorphism over $g^{-1}\left(\mathbf{R}^{m} \times F \times A\right)$.

Let $\Theta: \mathbf{R}^{m} \rightarrow \mathbf{R}^{m}$ be a radially defined homeomorphism such that $\Theta$ is supported on $B_{6.5}^{m} \backslash B_{1.5}^{m}$ and $\Theta\left(B_{6}^{m}\right)=B_{2}^{m}$. Then $\Theta$ induces a f.p. homeomorphism $\tilde{\Theta}: \mathbf{R}^{m} \times \Delta^{n} \rightarrow \mathbf{R}^{m} \times \Delta^{n}$ such that $\tilde{\Theta} \mid\left(\mathbf{R}^{m} \times \partial \Delta^{n}\right)=$ id and $\tilde{\Theta} \mid\left(\mathbf{R}^{m} \times\{t\}\right)=\Theta$ for each $t$ in $\Delta^{n} \backslash A$. On $\mathbf{R}^{m} \times A$ the action of $\Theta$ is phased out in such a way that $\tilde{\Theta}$ is supported on $\left(B_{6.5}^{m} \backslash B_{1.5}^{m}\right) \times \operatorname{int}\left(\Delta^{n}\right)$. Of course, there is also a f.p. isotopy $\tilde{\Theta}_{s}: \mathrm{id} \simeq \tilde{\Theta}, 0 \leq s \leq 1$, with the same support as $\tilde{\Theta}$. 
Now use $\left[\mathbf{H}_{1}\right]$ to find f.p. isotopies $\Gamma_{s}: M \rightarrow M$ and $\Lambda_{s}: N \rightarrow N$, $0 \leq s \leq 1$, such that

(1) $\Gamma_{0}=$ id and $\Lambda_{0}=\mathrm{id}$,

(2) $\Gamma_{s}$ is supported on $f^{-1}\left(\left(B_{6.6}^{m} \backslash B_{1.4}^{m}\right) \times F \times \operatorname{int}\left(\Delta^{n}\right)\right)$ and $\Lambda_{s}$ is supported on $g^{-1}\left(\left(B_{6.6}^{m} \backslash B_{1.4}^{m}\right) \times F \times \operatorname{int}\left(\Delta^{n}\right)\right)$,

(3) $(\pi \times \mathrm{id}) f \Gamma_{s}$ is $\varepsilon^{\prime}$-close to $\tilde{\Theta}_{s}(\pi \times \mathrm{id}) f$ and $(\pi \times \mathrm{id}) g \Lambda_{s}^{-1}$ is $\varepsilon^{\prime}$-close to $\tilde{\Theta}_{s}^{-1}(\pi \times \mathrm{id}) g$ where $\varepsilon^{\prime}>0$ is chosen small with respect to $\varepsilon$.

Then the desired map comes from setting $\tilde{h}=\Lambda_{1}^{-1} h \Gamma_{1}$ and the desired homotopy is given by $\Lambda_{s}^{-1} h \Gamma_{s}: h \simeq \tilde{h}, 0 \leq s \leq 1$.

Lemma 6.3 is used repeatedly to establish the following result. We continue to use the notation of Lemma 6.3.

LEMMA 6.4. For every $\varepsilon_{0}>0$ there exists a $\delta_{0}>0$ so that if $h: M \rightarrow$ $N$ is a f.p. map such that

(1) $h \mid \rho^{-1}\left(\partial \Delta^{n}\right)=\mathrm{id}$,

(2) $(\pi \times \mathrm{id}) g h$ is $\delta_{0}$-close to $f$,

(3) $h$ is a homeomorphism over $g^{-1}\left(B_{2}^{m} \times F \times \Delta^{n}\right)$, then $h$ is f.p. $((\pi \times \mathrm{id}) g)^{-1}\left(\varepsilon_{0}\right)$-homotopic rel $\rho^{-1}\left(\partial \Delta^{n}\right) \cup f^{-1}\left(B_{1}^{m} \times F \times\right.$ $\left.\Delta^{n}\right)$ to a homeomorphism $H: M \rightarrow N$.

Proof. Given $\varepsilon_{0}>0$ choose $\delta_{i}>0, i=1,2,3, \ldots$, so small that $\sum_{i=1}^{\infty} \delta_{i}<\varepsilon_{0}$ and so that $\delta_{i+1}$ is less than the $\delta\left(\delta_{i}\right)$ given by Lemma 6.3. By Lemma 6.3 there is a f.p. $((\pi \times \mathrm{id}) g)^{-1}\left(\delta_{1}\right)$-homotopy of $h$ to $h_{1}$ rel $\rho^{-1}\left(\partial \Delta^{n}\right) \cup f^{-1}\left(B_{1}^{m} \times F \times \Delta^{n}\right) \cup f^{-1}\left(\left(\mathbf{R}^{m} \backslash B_{6}^{m}\right) \times F \times \Delta^{n}\right)$ where $h_{1}$ is a homeomorphism over $g^{-1}\left(B_{5}^{m} \times F \times \Delta^{n}\right)$. Use Lemma 6.3 again to find a f.p. $((\pi \times \mathrm{id}) g)^{-1}\left(\delta_{2}\right)$-homotopy of $h_{1}$ to $h_{2}$ rel $\rho^{-1}\left(\partial \Delta^{n}\right) \cup f^{-1}\left(B_{3}^{m} \times F \times \Delta^{n}\right) \cup f^{-1}\left(\left(\mathbf{R}^{m} \backslash B_{8}^{m}\right) \times F \times \Delta^{n}\right)$ where $h_{2}$ is a homeomorphism over $g^{-1}\left(B_{7}^{m} \times F \times \Delta^{n}\right)$.

Continue in this manner to construct maps $h_{i}, i=1,2,3, \ldots$, so that $H=\lim _{i \rightarrow \infty} h_{i}$ is the desired homeomorphism. The reader should consult $\left[\mathbf{C}_{1}\right.$, pp. 327,328$]$ and $\left[\mathbf{H}_{2}\right.$, p. 626] for similar constructions.

Next we use Lemma 6.4 to obtain the following improvement of Lemma 6.2.

LEMMA 6.5. If $f: M \rightarrow \mathbf{R}^{m} \times F \times \Delta^{n}$ and $g: N \rightarrow \mathbf{R}^{m} \times F \times \Delta^{n}$ are $n$-simplices of $\mathscr{S}\left(\mathbf{R}^{m} \times F\right)$ such that $f=g$ over $\left(\mathbf{R}^{m} \times F \times \partial \Delta^{n}\right) \cup$ $\left(B_{2}^{m} \times F \times \Delta^{n}\right)$, then the conclusion of Lemma 6.2 holds. 
Proof. First find a f.p. homeomorphism $H: M \rightarrow N$ such that $H=$ id on $\rho^{-1}\left(\partial \Delta^{n}\right) \cup f^{-1}\left(B_{1.5}^{m} \times F \times \Delta^{n}\right)$ and $g H$ is f.p. $(\pi \times \text { id })^{-1}(\varepsilon)$ homotopic to $f$ rel $\rho^{-1}\left(\partial \Delta^{n}\right) \cup f^{-1}\left(B_{1.5}^{m} \times F \times \Delta^{n}\right)$ where $\varepsilon>0$ is as small as we want. This comes from the proof of Lemma 6.4. Note that Lemma 6.4 cannot be invoked directly because we do not start with a globally defined map $h: M \rightarrow N$. We do, however, have the identity

$$
\begin{aligned}
f^{-1}\left(\mathbf{R}^{m} \times F \times \partial \Delta^{n}\right) & \cup f^{-1}\left(B_{2}^{m} \times F \times \Delta^{n}\right) \\
& \rightarrow g^{-1}\left(\mathbf{R}^{m} \times F \times \partial \Delta^{n}\right) \cup g^{-1}\left(B_{2}^{m} \times F \times \Delta^{n}\right) .
\end{aligned}
$$

Since $H$ is defined by engulfing moves which repeatedly shrink and stretch radially, this clearly suffices.

Now $H$ can be used for the homeomorphism in the hypothesis of Lemma 6.2.

We are now ready for our first main result.

Proposition 6.6. $\gamma: \mathscr{S}\left(\mathbf{R}^{m} \times F\right) \rightarrow \mathscr{S} \mathscr{S}\left(\mathbf{R}^{m} \times F\right)$ is a homotopy equivalence.

Proof. We will show that $\gamma_{*}: \pi_{*} \mathscr{S}\left(\mathbf{R}^{m} \times F\right) \rightarrow \pi_{*} \mathscr{S} \mathcal{S}\left(\mathbf{R}^{m} \times F\right)$ is an isomorphism (for all $* \geq 0$ and for any choice of basepoint). Suppose that $n \geq 0$ and that we are given an $n$-simplex $\bar{f}$ of $\mathscr{S}\left(\mathbf{R}^{m} \times F\right)$ represented by $f: M \rightarrow T \times F \times \Delta^{n}$ where $T$ is a euclidean neighborhood of the origin in $\mathbf{R}^{m}$. Suppose that we are additionally given a union of $n+1(n-1)$-simplices of $\mathscr{S}\left(\mathbf{R}^{m} \times F\right)$ described by a map $g: N \rightarrow \mathbf{R}^{m} \times F \times \partial \Delta^{n}$ where $N \subset \mathbf{R}^{\infty} \times \partial \Delta^{n}$. That is, the restriction $g_{i}$ of $g$ to $N \cap\left(\mathbf{R}^{\infty} \times \partial_{i} \Delta^{n}\right)$ is an $(n-1)$-simplex of $\mathscr{S}\left(\mathbf{R}^{m} \times F\right)$ for $i=0,1, \ldots, n$. Let $f_{i}$ denote the restriction of $f$ over $\partial_{i} \Delta^{n}$ for $i=0,1, \ldots, n$. Finally, suppose that $\gamma\left(g_{i}\right)=\bar{f}_{i}$ for each $i=0,1, \ldots, n$, a condition that we write briefly as $\gamma(g)=\partial \bar{f}$. We will show that there is an $n$-simplex $\tilde{g}$ of $\mathscr{S}\left(\mathbf{R}^{m} \times F\right)$ such that $\partial \tilde{g}=g$ (i.e., $\partial_{i} \tilde{g}=g_{i}$ for $i=0,1, \ldots, n)$ and $\gamma(\tilde{g}) \simeq \bar{f}$ rel $\partial$. This (for all $n$ ) will imply that $\gamma_{*}$ is an isomorphism.

First note that by passing to a smaller neighborhood of the origin in $\mathbf{R}^{m}$, we may assume that $f=g$ over $T \times F \times \partial \Delta^{n}$. We may further assume that there is a homeomorphism $e: T \rightarrow \mathbf{R}^{m}$ such that $e \mid T_{0}=\mathrm{id}$ for some neighborhood $T_{0}$ of the origin.

Consider the composition

$$
\tilde{f}: M \stackrel{f}{\longrightarrow} T \times F \times \Delta^{n} \stackrel{e \times i d}{\longrightarrow} \mathbf{R}^{m} \times F \times \Delta^{n} .
$$


It is clear that $\tilde{f}$ is an $n$-simplex of $\mathscr{S}\left(\mathbf{R}^{m} \times F\right)$ and, since $\tilde{f}=f$ over $T_{0} \times F \times \Delta^{n}$, that $\gamma(\tilde{f})=\bar{f}$. It also follows that $\tilde{f}=g$ over $T_{0} \times F \times \partial \Delta^{n}$.

Now $\tilde{g}$ is easily constructed by using Lemma 6.5 to build a collar between $\partial \tilde{f}$ and $g$. This must be done inductively, working upward through the dimensions of the simplices of $\partial \Delta^{n}$.

We now proceed to globalize this result. We begin by defining the semi-simplicial complex $\mathscr{G} \tau \mathscr{S}(p: E \rightarrow B)$, the space of germs of tangentially controlled structures. A typical $n$-simplex of $\mathscr{G} \tau \mathscr{S}(p: E \rightarrow B)$ is an equivalence class $\bar{f}$ represented by a diagram

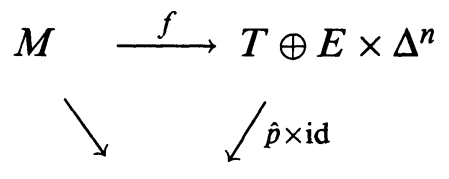

$$
B \times \Delta^{n}
$$

where

(1) $T$ is a tangent bundle neighborhood about $\Delta B$ in $B \times B$ with $p_{1}: T \rightarrow B$ the projection onto the first factor,

(2) $\hat{p}: T \oplus E \rightarrow B$ is the Whitney sum of the bundles $p_{1}: T \rightarrow B$ and $p: E \rightarrow B$,

(3) $M \subset \mathbf{R}^{s} \times B \times \Delta^{n} \subset \mathbf{R}^{\infty} \times B \times \Delta^{n}$ for some $s$ so that the projection $\mathbf{R}^{\infty} \times B \times \Delta^{n} \rightarrow B \times \Delta^{n}$ restricts to a fibering $M \rightarrow B \times \Delta^{n}$ with open $k$-manifold fibers,

(4) $f$ is fiber preserving over $B \times \Delta^{n}$ (which of course means that the diagram above commutes),

(5) for each $(b, t)$ in $B \times \Delta^{n}$, the map $f \mid: M_{(b, t)} \rightarrow \hat{p}^{-1}(b) \times\{t\}$ is a $\pi_{b}^{-1}(\alpha)$-equivalence for each open cover $\alpha$ of $p_{1}^{-1}(b)$ where $\pi_{b}$ : $\hat{p}^{-1}(b)=p_{1}^{-1}(b) \times p^{-1}(b) \rightarrow p_{1}^{-1}(b)$ denotes projection and $M_{(b, t)}=$ $M \cap \mathbf{R}^{\infty} \times\{(b, t)\}$.

Another such diagram

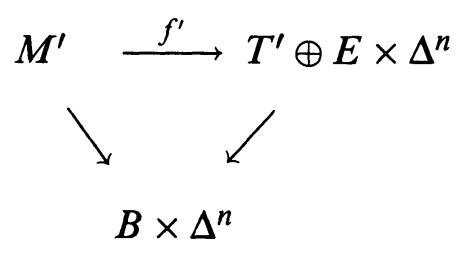

is equivalent to $f$ provided there is an open neighborhood $T^{\prime \prime}$ of $\Delta B$ in $B \times B$ such that $T^{\prime \prime} \subset T \cap T^{\prime}$ and $f=f^{\prime}$ over $T^{\prime \prime} \oplus E \times \Delta^{n}$. Of course, we could equivalently require that $T^{\prime \prime}$ be a tangent bundle neighborhood.

It is clear that $\mathscr{G} \tau \mathscr{S}(p: E \rightarrow B)$ can also be described as a space of cross-sections in the same manner that $\tau \mathscr{S}(p: E \rightarrow B)$ is the space 
of cross-sections of $\tilde{p}: \tilde{E} \rightarrow B$. To do this, proceed as in the last section. The group Sing $G$ acts on $\mathscr{G} \tau \mathscr{S}\left(\mathbf{R}^{m} \times F\right)$ and therefore we can construct a bundle $\bar{p}: \bar{E} \rightarrow B$, associated to $T B \oplus E$, with fiber $\mathscr{G S}\left(\mathbf{R}^{m} \times F\right)$. Then $\mathscr{G} \tau \mathscr{S}(p: E \rightarrow B)$ is the space of cross-sections of $\bar{p}: \bar{E} \rightarrow B$.

The homotopy equivalence $\gamma: \mathscr{S}\left(\mathbf{R}^{m} \times F\right) \rightarrow \mathscr{G S}\left(\mathbf{R}^{m} \times F\right)$ commutes with the actions of $\operatorname{Sing} G$. It follows that $\tilde{p}: \tilde{E} \rightarrow B$ and $\bar{p}: \bar{E} \rightarrow B$ are fiber homotopy equivalent, with the fiber homotopy equivalence being induced by $\gamma$. Now $\gamma$ also induces a map $\tilde{\gamma}: \tau \mathscr{S}(p: E \rightarrow B) \rightarrow \mathscr{G} \tau \mathscr{S}(p: E \rightarrow B)$ and the following result is immediate.

THEOREM 6.5. $\tilde{\gamma}: \tau \mathscr{S}(p: E \rightarrow B) \rightarrow \mathscr{G} \tau \mathscr{S}(p: E \rightarrow B)$ is a homotopy equivalence.

7. Tangentially controlled structures over open sets. In this section we will associate to each open subset $U$ of $B$ a semi-simplicial complex $\tau \mathscr{S}(U)$, the space of tangentially controlled structures over $U$. This is analogous to what was done in $\S 4$; however, the sheaf-theoretic properties will be obvious in the present situation.

Let $U$ be an open subset of $B$. Define the semi-simplicial complex $\tau \mathscr{S}(U)$ as follows. A typical $n$-simplex is an equivalence class $\bar{f}$ represented by a diagram

$$
\begin{aligned}
& M \stackrel{f}{\longrightarrow} T U \oplus p^{-1}(U) \times \Delta^{n} \\
& \searrow \quad / \hat{p} \times \text { id } \\
& U \times \Delta^{n}
\end{aligned}
$$

where

(1) $T U$ is a tangent bundle neighborhood about $\Delta U$ in $U \times B$ with $p_{1}: T U \rightarrow U$ the projection onto the first factor,

(2) $\hat{p}: T U \oplus p^{-1}(U) \rightarrow U$ is the Whitney sum of the bundles $p_{1}: T U \rightarrow U$ and $p \mid: p^{-1}(U) \rightarrow U$,

(3) $M \subset \mathbf{R}^{s} \times U \times \Delta^{n} \subset \mathbf{R}^{\infty} \times U \times \Delta^{n}$ for some $s$ so that the projection $\mathbf{R}^{\infty} \times U \times \Delta^{n} \rightarrow U \times \Delta^{n}$ restricts to a fibering $M \rightarrow U \times \Delta^{n}$ with open $k$-manifold fibers,

(4) $f$ is fiber preserving over $U \times \Delta^{n}$,

(5) For each $(b, t)$ in $U \times \Delta^{n}$, the map $f \mid: M_{(b, t)} \rightarrow \hat{p}^{-1}(b) \times\{t\}$ is a $\pi_{1}^{-1}(\alpha)$-equivalence for each open cover $\alpha$ of $p_{1}^{-1}(b)$ where $\pi_{b}$ : $\hat{p}^{-1}(b) \rightarrow \rho_{1}^{-1}(b)$ denotes projection and $M_{(b, t)}=M \cap \mathbf{R}^{\infty} \times\{(b, t)\}$. 
Another such diagram

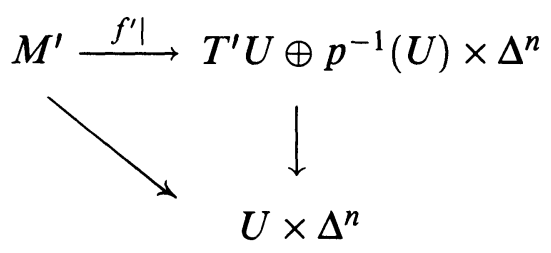

is equivalent to $f$ provided there is an open neighborhood $T^{\prime \prime}$ of $\Delta U$ in $U \times B$ such that $T^{\prime \prime} \subset T U \cap T^{\prime} U$ and $f=f^{\prime}$ over $T^{\prime \prime} \oplus E \times \Delta^{n}$.

Proposition 7.1. $\tau \mathscr{S}(p: E \rightarrow B)$ is homotopy equivalent to $\tau \mathscr{S}(B)$.

Proof. It is clear from the definitions that $\mathscr{G} \tau \mathscr{S}(p: E \rightarrow B)=$ $\tau \mathscr{S}(B)$. The result therefore follows from Theorem 6.5 .

If $V \subset U$ are open subsets of $B$, then there is an obviously defined restriction map $r: \tau \mathscr{S}(U) \rightarrow \tau \mathscr{S}(V)$. In the usual way we can define $\tau \mathscr{S}(X)$ for any compact subset of $B$ by passing to germs (i.e., by taking direct limits).

Recall from the discussion of the previous section that $\mathscr{G} \tau \mathscr{S}(p: E \rightarrow B$ ) (which is the same complex as $\tau \mathscr{S}(B)$ ) is just the space of cross-sections of the bundle $\bar{p}: \bar{E} \rightarrow B$. It is then clear that $\tau \mathscr{S}(U)$ is the space of cross-sections of the bundle $\bar{p} \mid: \bar{p}^{-1}(U) \rightarrow U$ and that the restriction $r: \tau \mathscr{S}(U) \rightarrow \tau \mathscr{S}(V)$ corresponds to restricting cross-sections.

Because of this cross-section interpretation of $\tau \mathscr{S}(U)$, the proof of the following proposition is rather obvious. This is remarkably different from the situation in $\S 4$.

Proposition 7.2. The functor $\tau \mathscr{S}$ satisfies the following sheaftheoretic conditions:

(1) If $X$ and $Y$ are compact subsets of $B$ then the following diagram of restriction maps is a fiber product

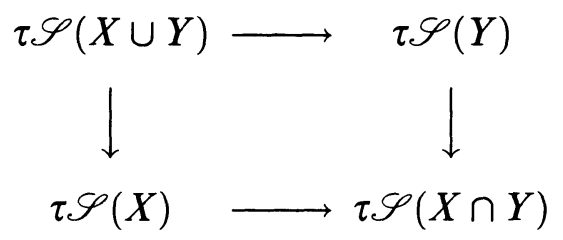

(2) If $Y \subset X$ is a compact pair in $B$, then the restriction $r: \tau \mathscr{S}(X) \rightarrow$ $\tau \mathscr{S}(Y)$ is a Kan fibration. 
(3) If $X$ is a simplex in $B$ (linear in some chart) and $x \in X$, then the restriction $r: \tau \mathscr{S}(X) \rightarrow \tau \mathscr{S}(x)$ is a homotopy equivalence.

8. The differential. $d: \mathscr{S}(U) \rightarrow \tau \mathscr{S}(U)$. In this section we will define and study a map $d: \mathscr{S}(U) \rightarrow \tau \mathscr{S}(U)$, called the differential over $U$. In the next section we will prove that $d: \mathscr{S}(B) \rightarrow \tau \mathscr{S}(B)$ is a homotopy equivalence.

Before we commence with the definition of the differential, we will fix some notation. Recall (from $\S 5$ ) that we have a fixed topological tangent bundle $p_{1}: T B \rightarrow B$ with zero section $\Delta B \subset T B \subset B \times B$. Now choose a homotopy $r_{t}: T B \rightarrow T B, 0 \leq t \leq 1$, such that

(1) $r_{0}=\mathrm{id}$,

(2) $r_{1}(T B)=\Delta B$,

(3) $r_{t} \mid \Delta B=$ id for each $t$,

(4) $p_{1} r_{t}=p_{1}$ for each $t$.

Since id $\times p: B \times E \rightarrow B \times B$ is a bundle projection, we can restrict over $T B$ to get a bundle projection $(\mathrm{id} \times p) \mid:(\mathrm{id} \times p)^{-1}(T B) \rightarrow T B$. Thus, we can use the homotopy lifting property to find a homotopy $\tilde{r}_{t}:(\mathrm{id} \times p)^{-1}(T B) \rightarrow(\mathrm{id} \times p)^{-1}(T B), 0 \leq t \leq 1$, such that

(1) $\tilde{r}_{0}=\mathrm{id}$,

(2) $(\mathrm{id} \times p) \tilde{r}_{t}=r_{t}(\mathrm{id} \times p)$ for each $t$,

(3) $\tilde{r}_{t} \mid(\mathrm{id} \times p)^{-1}(\Delta B)=\mathrm{id}$ for each $t$.

It follows that

(4) $\tilde{r}_{1}(\mathrm{id} \times p)^{-1}(T B) \subset(\mathrm{id} \times p)^{-1}(\Delta B)$,

(5) $p_{1}(\mathrm{id} \times p) \tilde{r}_{t}=p_{1}(\mathrm{id} \times p)$ for each $t$.

The definition of $d$ will depend (up to homotopy) on these choices for $r_{t}$ and $\tilde{r}_{t}$.

Let $U$ be the interior of a compact codimension 0 submanifold of $B$. Thus, $\mathscr{S}(U)$ is defined. We now proceed to define the differential $d: \mathscr{S}(U) \rightarrow \tau \mathscr{S}(U)$. Fix a tangent bundle neighborhood $T U$ about $\Delta U$ in $U \times U$ with $p_{1}: T U \rightarrow U$ projection onto the first factor. We may assume that $T U$ is sufficiently close to $\Delta U$ that $T U \subset T B$.

Form the Whitney sum $\hat{p}: T U \oplus p^{-1}(U) \rightarrow U$ of the bundles $p_{1}:$ $T U \rightarrow U$ and $p \mid: p^{-1}(U) \rightarrow U$.

Let $\mathbf{R}^{\infty} \times \Delta^{n} \supset M \stackrel{f}{\longrightarrow} p^{-1}(U) \times \Delta^{n}$ be an $n$-simplex of $\mathscr{S}(U)$. Consider the composition

$$
a: U \times M \stackrel{\text { id } \times f}{\longrightarrow} U \times p^{-1}(U) \times \Delta^{n} \stackrel{\text { id } \times p \times \text { id }}{\longrightarrow} U \times U \times \Delta^{n} .
$$

Let $\hat{M}=a^{-1}\left(T U \times \Delta^{n}\right)$. Note that $\hat{M} \subset U \times M \subset U \times \mathbf{R}^{\infty} \times \Delta^{n}$ and let $q: \hat{M} \rightarrow U \times \Delta^{n}$ denote the restriction of the projection. 
LEMMA 8.1. $q: \hat{M} \rightarrow U \times \Delta^{n}$ is a bundle projection.

Proof. We have a commuting diagram

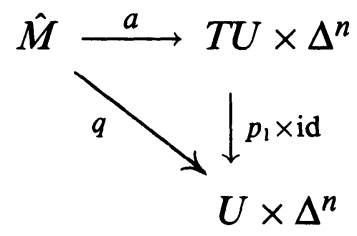

where

(1) $p_{1} \times$ id is a bundle projection with $\mathbf{R}^{m}$ fiber,

(2) $q$ is a submersion such that $q^{-1}(x)$ is an open $k$-manifold $(k \geq 5)$ for each $x$ in $U \times \Delta^{n}$,

(3) $a \mid: q^{-1}(x) \rightarrow\left(p_{1} \times \mathrm{id}\right)^{-1}(x)$ is an approximate fibration for each $x$ in $U \times \Delta^{n}$.

Using these three facts, it is easy to use the argument of Lemma 4.1 to deduce that $q$ is a bundle projection.

Now consider the following diagram:

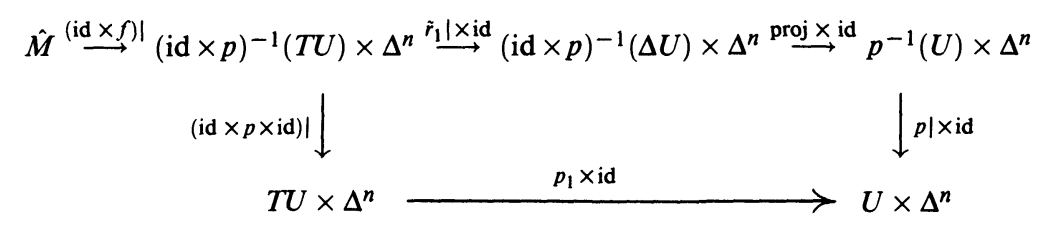

Perhaps the only map in this diagram which needs explanation is proj: $(\mathrm{id} \times p)^{-1}(\Delta U) \rightarrow p^{-1}(U)$. Here id $\times p: U \times p^{-1}(U) \rightarrow U \times U$ so that (id $\times p)^{-1}(\Delta U) \subset U \times p^{-1}(U)$. Thus, the map in question, proj, is just projection onto the second factor. With this understanding, it is easy to see that the square in the diagram above is commutative.

Now define $d_{1} f: \hat{M} \rightarrow T U \times \Delta^{n}$ by setting $d_{1} f=($ id $\times p \times$ id $)($ id $\times f) \mid$. And define $d_{2} f: \hat{M} \rightarrow p^{-1}(U) \times \Delta^{n}$ by setting $d_{2} f=(\operatorname{proj} \times \mathrm{id})\left(\tilde{r}_{1} \mid \times\right.$ id) $(\mathrm{id} \times f) \mid$.

After identifying $\left(T U \times \Delta^{n}\right) \oplus\left(p^{-1}(U) \times \Delta^{n}\right)$ with $\left(T U \oplus p^{-1}(U)\right) \times \Delta^{n}$, the commutativity of the diagram above says that $\left(d_{1} f, d_{2} f\right): \hat{M} \rightarrow$ $T U \times \Delta^{n} \times p^{-1}(U) \times \Delta^{n}$ defines a map $d f: \hat{M} \rightarrow\left(T U \oplus p^{-1}(U)\right) \times \Delta^{n}$.

Now consider the diagram

$$
U \times \mathbf{R}^{\infty} \times \Delta^{n} \supset \hat{M} \stackrel{d f}{\longrightarrow} T U \oplus p^{-1}(U) \times \Delta^{n}
$$


LEMMA 8.2. This diagram represents an $n$-simplex in $\tau \mathscr{S}(U)$.

Proof. First note that the fact that $\hat{M}$ is in $U \times \mathbf{R}^{\infty} \times \Delta^{n}$ instead of $\mathbf{R}^{\infty} \times U \times \Delta^{n}$ is no real problem.

Second note that the diagram commutes. This is seen by observing that $q=\left(p_{1} \times \mathrm{id}\right) d_{1} f$ by definition and that $(\hat{p} \times \mathrm{id}) d f=\left(p_{1} \times \mathrm{id}\right) d_{1} f$ by the nature of the Whitney sum.

Now, thanks to Lemma 8.1, it only remains to check that each "slice" of $d f$ (over an element of $U \times \Delta^{n}$ ) is the right kind of homotopy equivalence (as required by the definition in $\S 7$ ). For this, it suffices to show that each slice of $d_{1} f$ is an approximate fibration and that each slice of $d_{2} f$ is a homotopy equivalence.

To this end, note that if $(b, t)$ is in $U \times \Delta^{n}$, then

$$
q^{-1}(b, t)=\{b\} \times f^{-1}\left(p^{-1} p_{1}^{-1}(b) \times\{t\}\right) \subset U \times M .
$$

And $d_{1} f \mid q^{-1}(b, t)$ is given by the composition

$$
\begin{aligned}
\{b\} \times f^{-1}\left(p^{-1} p_{1}^{-1}(b) \times\{t\}\right) \stackrel{(\mathrm{id} \times f) \mid}{\longrightarrow} & \{b\} \times p^{-1} p_{1}^{-1}(b) \times\{t\} \\
& \stackrel{(\mathrm{id} \times p \times \mathrm{id}) \mid}{\longrightarrow}\{b\} \times p_{1}^{-1}(b) \times\{t\} .
\end{aligned}
$$

It follows that $d_{1} f \mid q^{-1}(b, t)$ is the approximate fibration $(p \times$ id $) f$ restricted over the open set $p_{1}^{-1}(b) \subset U$. Thus, $d_{1} f \mid q^{-1}(b, t)$ is an approximate fibration as required.

Now $d_{2} f \mid q^{-1}(b, t)$ is the composition

$$
\begin{gathered}
\{b\} \times f^{-1}\left(p^{-1} p_{1}^{-1}(b) \times\{t\}\right) \stackrel{(\mathrm{id} \times f) \mid}{\longrightarrow}\{b\} \times p^{-1} p_{1}^{-1}(b) \times\{t\} \stackrel{\left(\tilde{r}_{1} \mid \times \mathrm{id}\right)}{\longrightarrow} \mid \\
\{b\} \times p^{-1}(b) \times\{t\} \stackrel{\text { proj }}{\longrightarrow} p^{-1}(b) \times\{t\} .
\end{gathered}
$$

The first map $(\operatorname{id} \times f) \mid$ is a homotopy equivalence because it is the restriction over the open set $p^{-1}\left(p_{1}^{-1}(b)\right)$ of the map $f$ which is a $p^{-1}(\varepsilon)$-equivalence for every $\varepsilon>0$.

Is it not hard to see that the second map $\left(\tilde{r}_{1} \mid \times\right.$ id $) \mid$ in the composition is also a homotopy equivalence. It follows that $d_{2} f \mid q^{-1}(b, t)$ is a homotopy equivalence as required.

We have therefore succeeded in defining a semi-simplicial map $d$ : $\mathscr{S}(U) \rightarrow \tau \mathscr{S}(U)$. Since it is easy to see that $d$ commutes with restrictions, we have defined $d: \mathscr{S}(X) \rightarrow \tau \mathscr{S}(X)$ whenever $X$ is a compact subset of $B$.

The goal for the remainder of this section is to prove Theorem 8.3 below, which says that $d: \mathscr{S}(x) \rightarrow \tau \mathscr{S}(x)$ is a homotopy equivalence 
for each point $x$ in $B$. The proof will rely on the local triviality of bundles and we will need reinterpretations of the complexes $\mathscr{S}(x)$ and $\tau \mathscr{S}(x)$.

We begin by defining a semi-simplicial complex $\tau \mathscr{S}^{\prime}(x)$ as follows. A typical $n$-simplex is represented by a diagram

where:

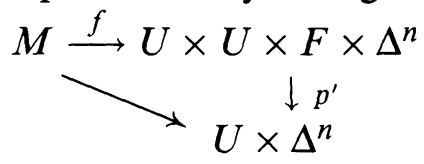

(1) $U$ is an open subset of $B$ containing $x$ such that $U$ is homeomorphic to $\mathbf{R}^{m}$ and $U \times U \subset T B$,

(2) $p^{\prime}$ is projection onto the first and fourth factors,

(3) $M \subset \mathbf{R}^{s} \times U \times \Delta^{n} \subset \mathbf{R}^{\infty} \times U \times \Delta^{n}$ for some $s$ so that the projection $\mathbf{R}^{\infty} \times U \times \Delta^{n} \rightarrow U \times \Delta^{n}$ restricts to a fibering $M \rightarrow U \times \Delta^{n}$ with open $k$-manifold fibers,

(4) $f$ is fiber preserving over $U \times \Delta^{n}$,

(5) for each $(b, t)$ in $U \times \Delta^{n}, f \mid: M_{(b, t)} \rightarrow\{b\} \times U \times F \times\{t\}$ is a $\pi^{-1}(\alpha)$-equivalence for every open cover $\alpha$ of $U$, where $\pi: U \times F \rightarrow U$ denotes projection and $M_{(b, t)}=M \cap \mathbf{R}^{\infty} \times\{(b, t)\}$.

Another such diagram

$$
\begin{gathered}
M^{\prime} \stackrel{f^{\prime}}{\longrightarrow} V \times V \times F \times \Delta^{n} \\
\searrow \underset{ }{\searrow} \times \Delta^{n}
\end{gathered}
$$

is equivalent to $f$ provided there exists an open subset $W$ of $B$ such that $x \in W \subset U \cap V$ and $f=f^{\prime}$ over $W \times W \times F \times \Delta^{n}$.

There is an isomorphism $t_{1}: \tau \mathscr{S}(x) \rightarrow \tau \mathscr{S}^{\prime}(x)$ which we now describe. An $n$-simplex of $\tau \mathscr{S}(x)$ is represented by an $n$-simplex of $\tau \mathscr{P}(U)$, where $U$ is an open subset of $B$ containing $x$, which is represented by a diagram

$$
\begin{gathered}
M \stackrel{f}{\longrightarrow} T U \oplus p^{-1}(U) \times \Delta^{n} \\
\searrow \\
U \times \Delta^{n}
\end{gathered}
$$

We may assume that $U$ is homeomorphic to $\mathbf{R}^{m}$ and that $T U=U \times$ $U \subset T B$. Let $h: U \times F \rightarrow p^{-1}(U)$ be a trivializing homeomorphism for $p \mid: p^{-1}(U) \rightarrow U$. This induces a trivializing homeomorphism $\tilde{h}$ : $U \times U \times F \rightarrow T U \oplus p^{-1}(U)$ defined by $\tilde{h}\left(u_{1}, u_{2}, y\right)=\left(u_{1}, u_{2}\right) \oplus h\left(u_{1}, y\right)$. Consider the diagram

$$
\begin{gathered}
M \stackrel{f}{\longrightarrow} T U \oplus p^{-1}(U) \times \Delta^{n} \stackrel{\tilde{h}^{-1} \times \mathrm{id}}{\longrightarrow} U \times U \times F \times \Delta^{n} \\
\searrow \\
U \times \Delta^{n}
\end{gathered}
$$


This represents an $n$-simplex of $\tau \mathscr{S}^{\prime}(X)$ which we denote by $t_{1} f$. It is clear that $t_{1}: \tau \mathscr{S}(x) \rightarrow \tau \mathscr{S}^{\prime}(x)$ is an isomorphism.

In a similar manner we define a semi-simplicial complex $\mathscr{S}^{\prime}(x)$. It has a typical $n$-simplex represented by $f: M \rightarrow U \times F \times \Delta^{n}$ where:

(1) $U$ is an open subset of $B$ containing $x$ such that $U$ is homeomorphic to $\mathbf{R}^{m}$,

(2) $M \subset \mathbf{R}^{s} \times \Delta^{n} \subset \mathbf{R}^{\infty} \times \Delta^{n}$ for some $s$ so that the projection $\mathbf{R}^{\infty} \times \Delta^{n} \rightarrow \Delta^{n}$ restricts to a fibering $\rho: M \rightarrow \Delta^{n}$ with open $k$-manifold fiber,

(3) $f$ is fiber preserving over $\Delta^{n}$,

(4) $f \mid: M_{t} \rightarrow U \times F \times\{t\}$ is a $\pi^{-1}(\alpha)$-equivalence for every open cover $\alpha$ of $U$, where $\pi: U \times F \rightarrow U$ is projection and $M_{t}=M \cap \mathbf{R}^{\infty} \times$ $\{t\}$, and each $t$ in $\Delta^{n}$.

Another such situation $f^{\prime}: M^{\prime} \rightarrow V \times F \times \Delta^{n}$ is equivalent to $f$ provided there exists an open subset $W$ of $B$ such that $x \in W \subset U \cap V$ and $f=f^{\prime}$ over $W \times F \times \Delta^{n}$.

As before there is an isomorphism $t_{2}: \mathscr{S}(x) \rightarrow \mathscr{S}^{\prime}(x)$ induced by the trivializing homeomorphism $h: U \times F \rightarrow p^{-1}(U)$.

We now want to understand the composition $d^{\prime}: \mathscr{S}^{\prime}(x) \stackrel{t_{2}^{-1}}{\longrightarrow} \mathscr{S}(x)$ $\stackrel{d}{\longrightarrow} \tau \mathscr{S}(x) \stackrel{t_{1}}{\longrightarrow} \tau \mathscr{S}^{\prime}(x)$. If $f: M \rightarrow U \times F \times \Delta^{n}$ represents an $n$ simplex of $\mathscr{S}^{\prime}(x)$, then one can trace through the definitions to see that $d^{\prime} f$ is represented by

$$
\begin{gathered}
U \times M \stackrel{d^{\prime} f}{\longrightarrow} U \times U \times F \times \Delta^{n} \\
\operatorname{id} \times \rho \searrow{ }^{\prime} \\
U \times \Delta^{n}
\end{gathered}
$$

where $d^{\prime} f(u, y)=\left(u, \operatorname{proj}_{U} f(y), \operatorname{proj}_{F} s_{1}\left(u, \operatorname{proj}_{U \times F} f(y)\right), \rho(y)\right)$. Here $s_{t}: U \times F \rightarrow U \times U \times F, 0 \leq t \leq 1$, is a homotopy such that

(1) $s_{t}$ is fiber preserving over the first $U$ factor for each $t$,

(2) $s_{0}=\mathrm{id}$,

(3) $s_{t} \mid \Delta U \times F=$ id for each $t$,

(4) $s_{1}(U \times U \times F) \subset \Delta U \times F$.

Note that $s_{t}$ depends on $\tilde{r}_{t}$ and $h$. We would like to replace $s_{t}$ by a strong deformation retraction $\tilde{s}_{t}$ where $\tilde{s}_{1}: U \times U \times F \rightarrow U \times U \times F$ is given by $\tilde{s}_{1}\left(u_{1}, u_{2}, y\right)=\left(u_{1}, u_{1}, y\right)$. If we do this then we have a new map $\tilde{d}: \mathscr{S}^{\prime}(x) \rightarrow \tau \mathscr{S}^{\prime}(x)$ which has the appealing form $\tilde{d} f=\operatorname{id}_{U} \times f$. Using the fact that the space of strong deformation retractions is path 
connected, it is easy to construct a homotopy $d^{\prime} \simeq \tilde{d}$. We are now ready for the result.

THEOREM 8.3. $d: \mathscr{S}(x) \rightarrow \tau \mathscr{S}(x)$ is a homotopy equivalence for each point $x$ in $B$.

Proof. By the remarks above it suffices to show tht $\tilde{d}: \mathscr{S}^{\prime}(x) \rightarrow$ $\tau \mathscr{S}^{\prime}(x)$ is a homotopy equivalence. For this we will show that $\tilde{d}_{*}$ : $\pi_{*} \mathscr{S}^{\prime}(x) \rightarrow \pi_{*} \tau \mathscr{S}^{\prime}(x)$ is an isomorphism. Clearly, $\tilde{d}_{*}$ is a monomorphism.

To see that $\tilde{d}_{*}$ is surjective, let $g: U \times M \rightarrow U \times U \times F \times \Delta^{n}$ represent a class $[g]$ in $\pi_{*} \tau \mathscr{S}^{\prime}(x)$. Then $g \mid:\{x\} \times M \rightarrow\{x\} \times U \times F \times \Delta^{n}$ represents a class $[g \mid]$ in $\pi_{*} \mathscr{S}^{\prime}(x)$. An easily constructed deformation of $g$ to $g \mid \times$ id near $x$ shows that $\tilde{d}_{*}([g \mid])=[g]$.

9. The main results. In this section we prove that $\mathscr{S}(p: E \rightarrow B)$ is homotopy equivalent to the space of cross-sections of a certain bundle over $B$. The proof is based on Gromov's immersion theoretic machine as delineated by Siebenmann in [KS, Essay V].

LEMMA 9.1. Let $X_{1}$ and $X_{2}$ be compacta in $B$ such that $d: \mathscr{S}\left(X_{i}\right) \rightarrow$ $\tau \mathscr{S}\left(X_{i}\right), i=1,2$, and $d: \mathscr{S}\left(X_{1} \cap X_{2}\right) \rightarrow \tau \mathscr{S}\left(X_{1} \cap X_{2}\right)$ are homotopy equivalences. Then $d: \mathscr{S}\left(X_{1} \cup X_{2}\right) \rightarrow \tau \mathscr{S}\left(X_{1} \cup X_{2}\right)$ is also a homotopy equivalence.

Proof. First recall that $r: \mathscr{S}\left(X_{2}\right) \rightarrow \mathscr{S}\left(X_{1} \cap X_{2}\right)$ and $r: \tau \mathscr{S}\left(X_{2}\right) \rightarrow$ $\tau \mathscr{S}\left(X_{1} \cap X_{2}\right)$ are Kan fibrations. Denote the fibers by $A_{1}$ and $A_{2}$ respectively. Consider the following commutative ladder whose rows are the fibration sequences of these fibrations and the vertical arrows are induced by $d$.

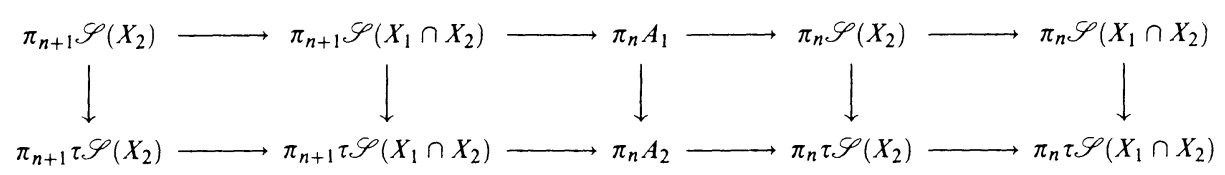

The five lemma implies that $d: A_{1} \rightarrow A_{2}$ is a homotopy equivalence.

We also have the fibrations $r: \mathscr{S}\left(X_{1} \cup X_{2}\right) \rightarrow \mathscr{S}\left(X_{1}\right)$ and $r$ : $\tau \mathscr{S}\left(X_{1} \cup X_{2}\right) \rightarrow \tau \mathscr{S}\left(X_{1}\right)$ whose fibers we denote by $B_{1}$ and $B_{2}$, respectively. 
The following diagram commutes.

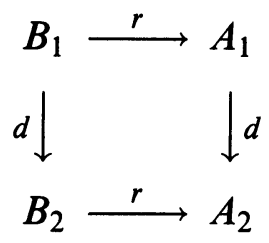

The right vertical arrow has just been shown to be a homotopy equivalence. The horizontal arrows are homotopy equivalences because

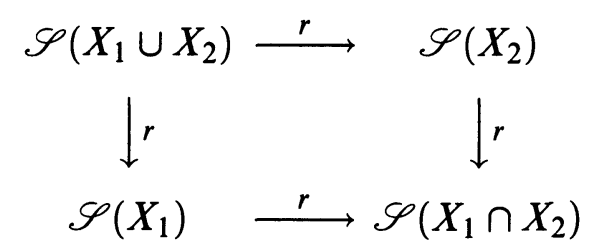

and

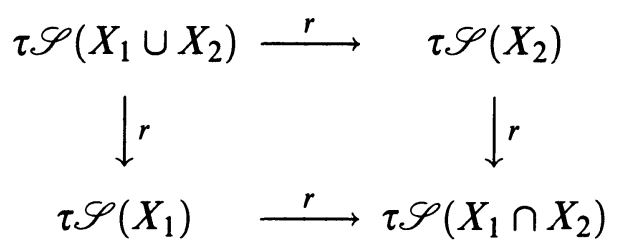

are fiber products. Thus, $d: B_{1} \rightarrow B_{2}$ is a homotopy equivalence.

Finally, consider the following commutative ladder whose rows are fibration sequences and vertical arrows are induced by $d$.

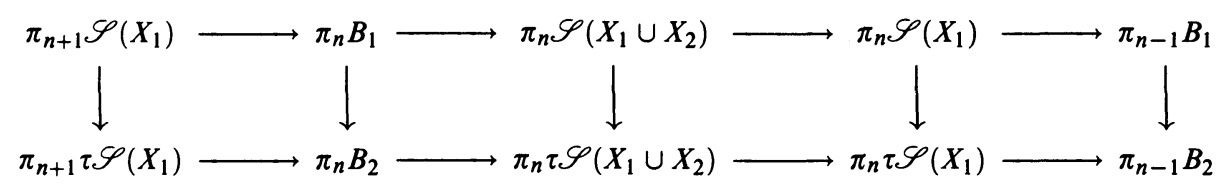

The five lemma shows that $d: \mathscr{S}\left(X_{1} \cup X_{2}\right) \rightarrow \tau \mathscr{S}\left(X_{1} \cup X_{2}\right)$ is a homotopy equivalence.

THEOREM 9.2. $d: \mathscr{S}(B) \rightarrow \tau \mathscr{S}(B)$ is a homotopy equivalence.

Proof. The proof is by "handle induction."

Step 1. If $X \subset B$ is a simplex (linear in some chart), then $d$ : $\mathscr{S}(X) \rightarrow \tau \mathscr{S}(X)$ is a homotopy equivalence.

Choose $x$ in $X$ and consider the commutative diagram

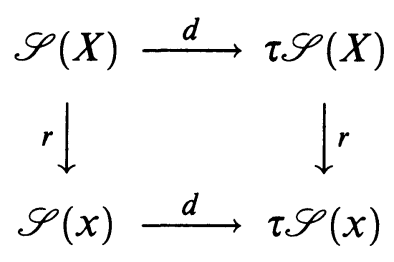


The vertical arrows are homotopy equivalences by Proposition 4.5 and Proposition 7.2, respectively. The bottom arrow is a homotopy equivalence by Theorem 8.3. Hence, the top arrow is a homotopy equivalence.

Step 2. If $X$ is a finite polyhedron in $B$ (linearly embedded in some chart), then $d: \mathscr{S}(X) \rightarrow \tau \mathscr{S}(X)$ is a homotopy equivalence.

This follows from Step 1, Lemma 9.1, and induction on the number of open cells of $X$.

Step 3. If $X$ is a compact subset of $B$ contained in some chart, then $d: \mathscr{S}(X) \rightarrow \tau \mathscr{S}(X)$ is a homotopy equivalence.

This follows because $X$ is the nested intersection of polyhedra for which Step 2 applies.

Step 4. $d: \mathscr{S}(B) \rightarrow \tau \mathscr{S}(B)$ is a homotopy equivalence.

This is because $B$ is the finite union of compacta for which Step 3 applies and whose pairwise intersections are also covered by Step 3. Thus, one uses induction, Step 4, and Lemma 9.1.

Corollary 9.3. $\mathscr{S}(p: E \rightarrow B)$ and $\tau \mathscr{S}(p: E \rightarrow B)$ are homotopy equivalent.

Proof. Recall from $\S 4$ that $\mathscr{S}(B)$ and $\mathscr{S}(p: E \rightarrow B)$ denote the same complex. And it follows from Proposition 7.1 that $\tau \mathscr{S}(B)$ and $\tau \mathscr{S}(p: E \rightarrow B)$ are homotopy equivalent. The result now follows from Theorem 9.2.

Corollary 9.4. $\mathscr{S}(p: E \rightarrow B)$ is homotopy equivalent to the space of cross-sections of the bundle $\tilde{p}: \tilde{E} \rightarrow B$ constructed in $\S 5$.

Proof. This follows from Corollary 9.3 and the definition of $\tau \mathscr{S}(p$ : $E \rightarrow B)$ in $\S 5$.

Note that Corollary 9.4 is just a restatement of Theorem 1 in the introduction.

\section{REFERENCES}

[AH] D. R. Anderson and W. C. Hsiang, The functors $K_{-\imath}$ and pseudo-isotopies of polyhedra, Ann. of Math., (2) 105 (1977), 201-223.

[C $\mathrm{C}_{1}$ T. A. Chapman, Approximation results in Hilbert cube manifolds, Trans. Amer. Math. Soc., 262 (1980), 303-334.

[C $\left.\mathrm{C}_{2}\right]$, Approximation results in topological manifolds, Mem. Amer. Math. Soc., 34 (1981), no. 251. 
[CF] T. A. Chapman and S. Ferry, Approximating homotopy equivalences by homeomorphisms, Amer. J. Math., 101 (1979), 583-607.

[CD] D. S. Coram and P. F. Duvall, Jr., Approximate fibrations, Rocky Mountain J. Math., 7 (1977), 275-288.

[EK] R. D. Edwards and R. C. Kirby, Deformations of spaces of imbeddings, Ann. of Math., (2) 93 (1971), 63-88.

[FH $\left.\mathrm{FH}_{1}\right]$ F. T. Farrell and W. C. Hsiang, Topological characterization of flat and almost flat Riemannian manifolds $M^{n}(n \neq 3,4)$, Amer. J. Math., 105 (1983), 641-672.

[FH $\mathrm{FH}_{2}$, A structure set analogue of Chapman-Ferry-Quinn theory, in Continua, Decompositions, Manifolds, Univ. of Texas Press, Austin, 1983.

$\left[\mathrm{H}_{1}\right] \quad$ C. B. Hughes, Approximate fibrations on topological manifolds, Michigan Math. J., 32 (1985), 167-183.

[H $\left.\mathrm{H}_{2}\right]$, Bounded homotopy equivalences of Hilbert cube manifolds, Trans. Amer. Math. Soc., 287 (1985), 621-643.

$\left[\mathrm{H}_{3}\right] \quad$ Delooping controlled pseudo-isotopies of Hilbert cube manifolds, to appear in Topology and its Appl.

[HTW] C. B. Hughes, L. R. Taylor and E. B. Williams, in preparation.

[KS] R. C. Kirby and L. C. Siebenmann, Foundational essays on topological manifolds, smoothings, and triangulations, Ann. of Math. Stud., 88, Princeton Univ. Press, Princeton, 1977.

[M] J. P. May, Simplicial Objects in Algebraic Topology, Van Nostrand-Reinhold, Princeton, 1967.

[Q1] F. Quinn, Ends of maps, I, Ann. of Math., (2) 110 (1979), 275-331.

[Q2] Ends of maps, II, Invent. Math. 68 (1982), 353-424.

[Q $\left.\mathrm{Q}_{3}\right]$, Ends of maps, III: dimensions 4 and 5, J. Differential Geometry, 17 (1982), 503-521.

[Q4] Ends of maps, IV: controlled pseudoisotopy, to appear in Amer. J. Math.

[Q5] Resolutions of homology manifolds, and the topological characterization of manifolds, Invent. Math., 72 (1983), 267-284.

[Q6] An obstruction to the resolution of homology manifolds, to appear in Invent. Math.

[Q7] Geometric algebra and ends of maps, preprint (1984).

[S] L. C. Siebenmann, Deformations of homeomorphisms on stratified sets, Comment. Math. Helv. 47 (1972), 123-153.

Received September 25, 1986 and in revised form August 21, 1987. Supported in part by NSF Grant DMS-8401570 and the University of Utah Research Fund.

VANDERBILT UNIVERSITY

NASHVILLE, TN 37235 



\section{PACIFIC JOURNAL OF MATHEMATICS}

\section{EDITORS}

V. S. VARADARAJAN

(Managing Editor)

University of California

Los Angeles, CA 90024

Herbert Clemens

University of Utah

Salt Lake City, UT 84112

R. FINN

Stanford University

Stanford, CA 94305
HERMANN FLASCHKA

University of Arizona

Tucson, AZ 85721

RAMESh A. GANGOLLI

University of Washington Seattle, WA 98195

VAUGHAN F. R. JONES

University of California

Berkeley, CA 94720
ROBION KIRBY

University of California

Berkeley, CA 94720

C. C. MOORE

University of California

Berkeley, CA 94720

HAROLD STARK

University of California, San Diego

La Jolla, CA 92093

\section{ASSOCIATE EDITORS}
R. AREnS
E. F. BECKENBACH
B. H. NEUMANN
F. WOLF
K. YOSHIDA (1906-1982)

\section{SUPPORTING INSTITUTIONS}

UNIVERSITY OF ARIZONA

UNIVERSITY OF OREGON

UNIVERSITY OF BRITISH COLUMBIA UNIVERSITY OF SOUTHERN CALIFORNIA

CALIFORNIA INSTITUTE OF TECHNOLOGY

UNIVERSITY OF CALIFORNIA

STANFORD UNIVERSITY

MONTANA STATE UNIVERSITY

UNIVERSITY OF HAWAII

UNIVERSITY OF NEVADA, RENO

UNIVERSITY OF TOKYO

NEW MEXICO STATE UNIVERSITY

UNIVERSITY OF UTAH

OREGON STATE UNIVERSITY

WASHINGTON STATE UNIVERSITY

UNIVERSITY OF WASHINGTON 


\section{Pacific Journal of Mathematics}

\section{Vol. 133, No. $1 \quad$ March, 1988}

John Anderson, Finitely generated algebras and algebras of solutions to partial differential equations $\ldots \ldots \ldots \ldots \ldots \ldots \ldots \ldots \ldots \ldots \ldots \ldots \ldots$

Junichi Aramaki, On an extension of the Ikehara Tauberian theorem . . . . . 13

Giacomo Monti Bragadin, Abstract Riemannian stratifications .......... 31

Lawrence James Brenton and Richard Hill, On the Diophantine equation

$1=\sum 1 / n_{i}+1 / \prod n_{i}$ and a class of homologically trivial complex

surface singularities .................................41

C. Bruce Hughes, Controlled homotopy topological structures $\ldots . \ldots \ldots \ldots 69$

Peter Wilcox Jones and Takafumi Murai, Positive analytic capacity but zero Buffon needle probability . ...........................999

Gary M. Lieberman, Hölder continuity of the gradient at a corner for the

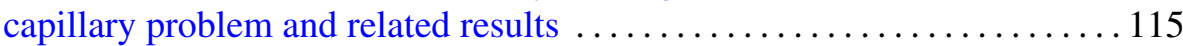

Feng Luo, Representing homology classes of $C \mathbf{P}^{2} \overline{C \mathbf{P}^{2}}$

Claudio Nebbia, Groups of isometries of a tree and the Kunze-Stein

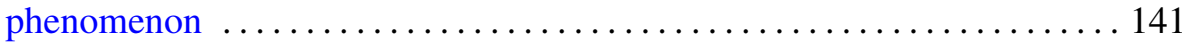

Stefan Richter, Unitary equivalence of invariant subspaces of Bergman and

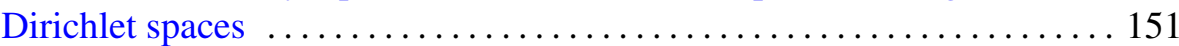

Paul Frederick Ringseth, The Selberg trace formula for groups without Eisenstein series ..................................... 157

Abderrazzak Sersouri, The Mazur property for compact sets $\ldots \ldots \ldots \ldots 185$ Alladi Sitaram, On an analogue of the Wiener Tauberian theorem for symmetric spaces of the noncompact type 